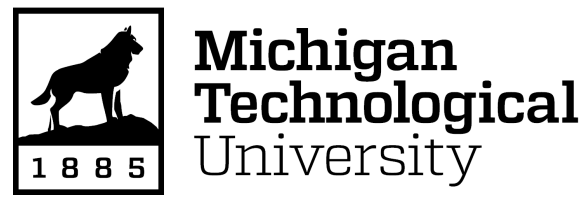

Michigan Technological University Digital Commons @ Michigan Tech

Dissertations, Master's Theses and Master's Reports

2017

\title{
Gamma/Hadron Separation for the HAWC Observatory
}

Michael J. Gerhardt

Michigan Technological University, mjgerhar@mtu.edu

Copyright 2017 Michael J. Gerhardt

\section{Recommended Citation}

Gerhardt, Michael J., "Gamma/Hadron Separation for the HAWC Observatory", Open Access Master's Thesis, Michigan Technological University, 2017.

https://doi.org/10.37099/mtu.dc.etdr/427

Follow this and additional works at: https://digitalcommons.mtu.edu/etdr

Part of the Multivariate Analysis Commons, and the Other Physics Commons 


\title{
GAMMA/HADRON SEPARATION FOR THE HAWC OBSERVATORY
}

\author{
By
}

Michael J. Gerhardt

\begin{abstract}
A THESIS
Submitted in partial fulfillment of the requirements for the degree of MASTER OF SCIENCE

In Physics

MICHIGAN TECHNOLOGICAL UNIVERSITY
\end{abstract}

2017

(C) 2017 Michael J. Gerhardt 

This thesis has been approved in partial fulfillment of the requirements for the Degree of MASTER OF SCIENCE in Physics.

Department of Physics

\author{
Thesis Advisor: $\quad$ Dr. Petra Huentemeyer \\ Committee Member: Dr. Brian Fick \\ Committee Member: Dr. David Nitz \\ Department Chair: Dr. Ravindra Pandey
}





\section{Contents}

List of Figures $\ldots \ldots \ldots \ldots \ldots \ldots \ldots \ldots \ldots \ldots$

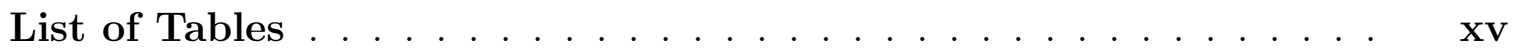

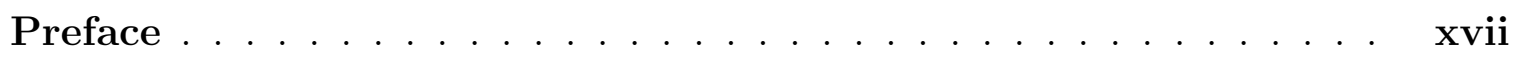

Acknowledgments ...................

Abstract ..........................

1 Introduction ........................... 1

1.1 Gamma-Ray Astrophysics . . . . . . . . . . . . . . . 1

1.2 Extensive Air Showers . . . . . . . . . . . . . . . 2

1.2.1 Gamma-Ray Induced Showers . . . . . . . . . . . 2

1.2.2 Cosmic-Ray Induced Showers _ . . . . . . . . . . 3

1.3 Detection with an EAS Array $\ldots \ldots \ldots \ldots \ldots$

1.3.1 Cherenkov Radiation . . . . . . . . . . . . . 5

$1.3 .2 \quad$ EAS Arrays $\ldots \ldots \ldots \ldots \ldots \ldots \ldots$

1.4 Multivariate Analysis . . . . . . . . . . . . . . . 8 8 
1.4.1 Decision Trees . . . . . . . . . . . . . . 9

1.4.2 Boosting methods ................ 11

2 HAWC Observatory ................... 15

2.1 Detector ....................... 15

2.2 Event Reconstruction . . . . . . . . . . . . . . . . . 16

2.2.1 Edge Finding . . . . . . . . . . . . . . 17

2.2 .2 Core Fit . . . . . . . . . . . . . . . . . . 19

2.2.3 Angular Fit . . . . . . . . . . . . . . . 22

2.3 Gamma/Hadron Separation . . . . . . . . . . . . . 23

2.3.1 PINCness and Compactness ............. 24

2.4 Calibration System . . . . . . . . . . . . . . 25

3 Calibration Procedure . . . . . . . . . . . . . . . . 29

3.1 Calibration Steps . . . . . . . . . . . . . . . . 29

3.2 Charge Calibration . . . . . . . . . . . . . 30

3.2.1 Occupancy method .................. 31

3.2.2 Charge Calibration Autocheck . . . . . . . . . 33

3.3 Timing Calibration .................... 34

3.3.1 Slewing Calibration ................ 35

3.3.1.1 Slewing Autocheck ............ 37

3.3.1.2 Distributions of Fit Parameters . . . . . . . 38

3.3.1.3 Modifid $\chi^{2}$ Distribution Check . . . . . . . 38 
3.3.2 Timing Pedestal Calibration . . . . . . . . . . . 42

3.3.2.1 Timing Autocheck . . . . . . . . . . 43

3.4 Zenith Alignment . . . . . . . . . . . . . . . . . . . . . 44

3.5 Diagnostic Checks . . . . . . . . . . . . . . 45

4 Gamma/Hadron Separation with Multivariable Analysis . . . . 47

4.1 TMVA Boosted Decision Trees . . . . . . . . . . . . . 48

4.1 .1 Training of BDTs . . . . . . . . . . . 48

4.1 .2 Testing of BDTs . . . . . . . . . . . 50

4.2 Analysis and Results of the Crab Nebula . . . . . . . . 52

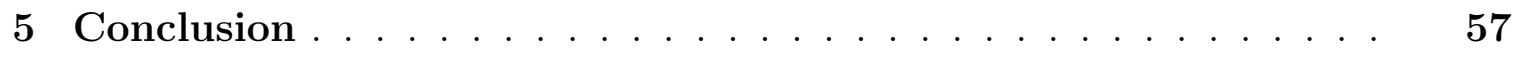

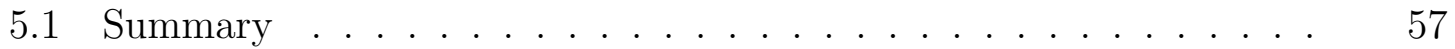

5.1 .1 Calibration . . . . . . . . . . . . 57

5.1.2 Gamma Hadron Separation with BDTs . . . . . . . . 58

5.2 Continuing Work $\ldots \ldots \ldots \ldots$

5.2 .1 Calibration Procedure Update . . . . . . . . . . . 59

5.2.2 Gamma Hadron Separation Optimization . . . . . . . . 59

References ....................... 61

A Plots from Multivariate Analysis . . . . . . . . . . . 65

A.1 Training and Testing Plots for bins $1-8 \ldots \ldots \ldots \ldots$

A.2 Crab Significance Maps for bins $4-8 \ldots \ldots \ldots \ldots$ 
B Letters of Permission . . . . . . . . . . . . . . . 72

B.1 Figure $1.2 \ldots \ldots \ldots \ldots \ldots$. . . . . . . . . . . . . . . . 72

B.2 Figure $1.4 \ldots \ldots \ldots \ldots \ldots$. . . . . . . . . . . . . . . . 72

B.3 Figure $2.1 \ldots \ldots \ldots \ldots$

B.4 Figure $2.2 \ldots \ldots \ldots \ldots \ldots$

B.5 Figure 2.3 , 2.5 . . . . . . . . . . . . . . . . . . . . 73

B.6 Figure $2.4 \ldots \ldots \ldots \ldots$

B.7 Figure $2.7 \ldots \ldots \ldots \ldots$

B.8 Figure $3.1,3.23 .3 \ldots \ldots \ldots \ldots$ 


\section{List of Figures}

1.1 Sketch of gamma ray induced air shower a resulting interactions . .

1.2 Diagram of proton induced shower entering the atmosphere producing initial secondary particles. These showers make up $90 \%$ of produced

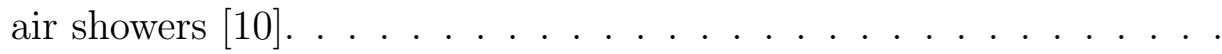

1.3 Sketch of electromagnetic shock front cause by Cherenkov radiation

1.4 Simulations of $10 \mathrm{TeV}$ proton (right) and photon (left) induced showers. Red is the electromagnetic portion (electrons, positrons, photons), blue is the hadronic component, and green is the muon component. CORSIKA software was used to make these plots [7] [3] . . . . .

1.5 Example decision tree with separation parameters being cut on at each level, where B, C, and P are properties of the events. . . . . . . 10

2.1 The HAWC Detector Array. . . . . . . . . . . . . . . 16

2.2 Tank schematic, showing PMT setup with optical fibers and diffuser $[10] \ldots \ldots \ldots \ldots \ldots \ldots \ldots$

2.3 High and Low threshold sample of signal (voltage) pulse and the edges produced by threshold crossing [24]. . . . . . . . . . . . 
2.4 Charge distribution as a function of the distance of the PMT location (location of the charge measurement) from the core. Showing best fit of gaussian-NKG function after the $\chi^{2}$ minimization (Blue) and the NKG fit using the core position from the gaussian-NKG function (Green) needed for determining the shower age and amplitude parameters [3]. 21

2.5 Example shower front hitting detector [24].......... 22

2.6 The gamma ray (top) and hadron (bottom) distributions of PINCness vs. Compactness for $f_{\text {hit }}$ bin $9 \ldots \ldots \ldots \ldots$

2.7 Layout of HAWC calibration system [14] . . . . . . . . . . . 28

3.1 Plot of $\log \left(N_{P E}\right)$ as a function of ToT counts for one PMT channel in the HAWC array [21]. . . . . . . . . . . . . . . . . 33

3.2 Charge distribution of PMT F8A [21]. . . . . . . . . . 34

3.3 Distributions of the SPE value (left) and the width of the SPE gaussian (right) for 8"(blue) and 10" (magenta) PMTs. The cut value lines for the 8" PMTs (red) and 10" PMTs (green) are also shown [21]. . . 35

3.4 Slewing curves of WCD H19, with fitting function. The two populations for each PMT are for the high and low threshold with the high threshold fit being above the low threshold fit. The A,B and D PMTs are 8" PMTs. The C PMT is the 10" PMT and installed at the center

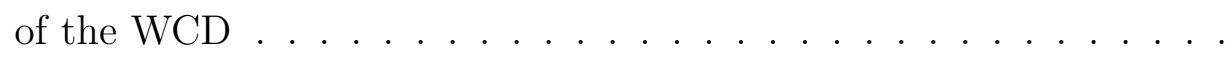


3.5 The distribution of the slewing fit parameters, $p_{0}$ (upper left), $p_{1}$ (upper right), $p_{2}$ (lower left), $p_{3}$ (lower right) for the low threshold fit. The green distribution contains all 1200 PMT IDs. The blue distribution contains all PMTs assigned the average curve, due to not being active at the time of calibration or not receiving laser light. The red distribution represents the channels known to be bad. . . . . . . .

3.6 The distribution of the slewing fit parameters, $p_{0}$ (upper left), $p_{1}$ (upper right), $p_{2}$ (lower left), $p_{3}$ (lower right) for the high threshold fit. The green distribution contains all 1200 PMT IDs. The blue distribution contains all PMTs assigned the average curve, due to not being active at the time of calibration or not receiving laser light. The red distribution represents the channels known to be bad.

3.7 Distributions of time residuals and time residual widths for $\mathrm{PE}>5$ including outlier PMTs that would be removed by flagged as bad by

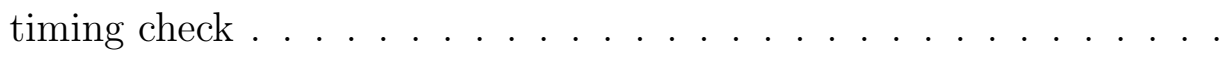

4.1 The response values, $y_{\text {boost }}$, of signal and background data for both the training and testing of the BDTs shown for $f_{\text {hit }}$ bin $9 . \ldots . .$.

4.2 Efficiency plot for both the signal and background data vs the response value, $y_{\text {boost }}$, for $f_{\text {hit }}$ bin $9 . \ldots \ldots \ldots$ 
4.3 The $f_{\text {hit }}$ bin 9 significance maps of the Crab Nebula for Nov-Dec 2015 for both the standard HAWC cuts (left) and the response value cut

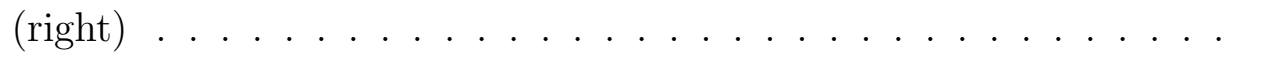

A.1 Response values for the BDTs for signal and background testing and training data (left) and effiecieny of the background and signal data vs response value(right) using $f_{\text {hit }}$ bin 1. . . . . . . . . . . . 65

A.2 Response values for the BDTs for signal and background testing and training data (left) and effiecieny of the background and signal data vs response value (right) using $f_{\text {hit }}$ bin $2 . \ldots . . . . . . . . .66$

A.3 Response values for the BDTs for signal and background testing and training data (left) and effiecieny of the background and signal data vs response value (right) using $f_{\text {hit }}$ bin $3 . \ldots . . . . . . . . .666$

A.4 Response values for the BDTs for signal and background testing and training data (left) and effiecieny of the background and signal data vs response value (right) using $f_{\text {hit }}$ bin $4 . \ldots . \ldots$. . . . . . 67

A.5 Response values for the BDTs for signal and background testing and training data (left) and effiecieny of the background and signal data vs response value (right) using $f_{\text {hit }}$ bin 5. . . . . . . . . . . 67

A.6 Response values for the BDTs for signal and background testing and training data (left) and effiecieny of the background and signal data vs response value (right) using $f_{\text {hit }}$ bin $6 . \ldots \ldots$. . . . . . . 67 
A.7 Response values for the BDTs for signal and background testing and training data (left) and effiecieny of the background and signal data vs response value (right) using $f_{\text {hit }}$ bin $7 . \ldots . \ldots . \ldots 6$

A.8 Response values for the BDTs for signal and background testing and training data (left) and effiecieny of the background and signal data vs response value (right) using $f_{\text {hit }}$ bin $8 . \ldots . . . . . . . . .668$

A.9 Significance maps of the Crab Nebula for $f_{\text {hit }}$ Bin 4 with standard PINCness and compactness cuts (left) and new BDT response value cuts (right) ............................ 69

A.10 Significance maps of the Crab Nebula for $f_{\text {hit }}$ Bin 5 with standard PINCness and compactness cuts (left) and new BDT response value cuts (right) ........................... 69

A.11 Significance maps of the Crab Nebula for $f_{\text {hit }}$ Bin 6 with standard PINCness and compactness cuts (left) and new BDT response value cuts (right) ....................... 70

A.12 Significance maps of the Crab Nebula for $f_{\text {hit }}$ Bin 7 with standard PINCness and compactness cuts (left) and new BDT response value cuts (right) ....................... 70

A.13 Significance maps of the Crab Nebula for $f_{\text {hit }}$ Bin 8 with standard PINCness and compactness cuts (left) and new BDT response value

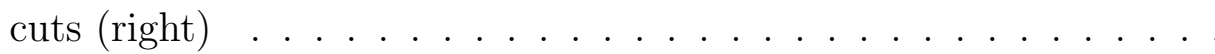





\section{List of Tables}

$2.1 f_{h i t}$ bins where $f_{\text {hit }}$ is the percentage of properly calibrated PMTs participating in an event $[1] \ldots \ldots \ldots \ldots \ldots$

4.1 List of tree parameters, with the min node size being a percentage of initial input events in a tree. Each boosting method described in Ch. 1 is used. The leaf nodes are defined based upon their purity instead of given a simple $\pm 1 \ldots \ldots \ldots \ldots \ldots \ldots$

4.2 The gamma ray and hadron efficiencies and the maximum significance value for $f_{\text {hit }}$ bins 4-9 for both the standard PINCness and compactness cuts and the response value cut. The cut value for the response value yielding the maximum significance is also shown for each bin. The gamma and hadron efficiencies for the standard cuts are taken from the published HAWC analysis of the Crab Nebula [1] and the efficiencies for the response value are from the testing results. . . . . . . . 



\section{Preface}

High Altitude Water Cherenkov (HAWC) Gamma-Ray Observatory is operated by a collaboration of more than 100 scientists and engineers from institutions in the United States and Mexico. The procedure for the timing calibration of the HAWC Detector described in Chapter 3 was created by Hao Zhou [24] and further developed by me. My contributions to the timing calibration are the slewing and timing pedestal autochecks along with the zenith alignment correction and timing portion of the diagnostic checks. The study of applying a multivariate analysis to HAWC data described in Chapter 4 using open source software provided by CERN is my original work. 



\section{Acknowledgments}

Thanks to the family, friends, and colleagues for their help and patience. In particular my advisor Dr. Petra Huentemeyer for consistently pushing me, Dr. Hao Zhou for teaching me how HAWC is calibrated, Dr. Henrike Fleischhack for helping me better understand BDTs, and Dr. Hugo Alaya-Solares and Chad Brisbois for helping me with my many coding issues in ROOT and $\mathrm{C}++$. 



\section{Abstract}

The High-Altitude Water Cherenkov (HAWC) Observatory is a gamma-ray observatory sensitive to gamma rays from $100 \mathrm{GeV}$ to $100 \mathrm{TeV}$ with an instantaneous field of view of $\sim 2$ sr. It is located on the Sierra Negra plateau in Mexico at an elevation of 4,100 $\mathrm{m}$ and began full operation in March 2015. The purpose of the detector is to study relativistic particles that are produced by interstellar and intergalactic objects such as: pulsars, supernova remnants, molecular clouds, black holes and more. To achieve optimal angular resolution, energy reconstruction and cosmic ray background suppression for the extensive air showers detected by HAWC, good timing and charge calibration are crucial, as well as optimization of quality cuts on background suppression variables. Additions to the HAWC timing calibration, in particular automating the calibration quality checks and a new method for background suppression using a multivariate analysis are presented in this thesis. 



\section{Chapter 1}

\section{Introduction}

\subsection{Gamma-Ray Astrophysics}

Gamma-ray astrophysics covers a large photon energy range from $\mathrm{MeV}$ to $\mathrm{TeV}$ [2]. There are a variety of different processes that generate these high energy photons in space. The four main contributors are $\pi^{0}$ decay, synchrotron radiation, inverse compton scattering,xs and bremsstrahlung radiation. Both space and ground based detectors are used to detect these photons. Space detectors can detect photons of energies up to hundreds of GeVs [14]. Ground based detectors are required for the measurement of energies greater than this. The reason is that as energy increases, the flux for gamma and cosmic rays decreases according to a power law, requiring 
a large detector area. Because it is financially impractical to send up into space a detector of sufficient size, ground based detectors are used instead. There are two main types of ground based detectors for gamma-ray energies, Imaging Atmospheric Cherenkov Telescopes (IACTs) and Extensive Air Shower (EAS) arrays. IACTs have the advantage of higher angular resolution and a clear energy resolution, but have a limited field of view and detector uptime. EAS arrays have the advantage of a high duty cycle and large field of view and will be discussed in more detail in a following section.

\subsection{Extensive Air Showers}

\subsubsection{Gamma-Ray Induced Showers}

When a photon enters the atmosphere it will cause electron-positron pair production. Pair production is when an electron and positron are produced due to a very high energy photon interaction with a nucleus, where the nucleus absorbs most of some of the photon energy and the remaining photon energy is converted into an electron and positron. These particles will produce bremsstrahlung (German for breaking radiation) radiation, which is electromagnetic radiation caused by the loss of kinetic energy of a charged particle due to its deflection by another charged particle. The 
average energy loss rate of electrons due to bremsstrahlung radiation is

$$
-\left(\frac{d E_{e}}{d t}\right)=\left(\frac{c m_{p} n}{X_{0}}\right) E_{e}
$$

where $c$ is the speed of light, $m_{p}$ is the mass of a proton, $n$ is the number density of the medium, and $X_{0}$ is the radiation length or the average distance for an electron to lose all but $1 / e$ of its energy [3]. This distance is equal to $7 / 9$ of the mean free path for pair production [24]. The resulting photons will produce new electron positron pairs. This will continue and produce a cascade of secondary particles called an extensive air shower (EAS) [24]. A sketch of this electromagnetic cascade is shown in Fig. 1.1. The cascade continues until the average energy of the electrons and positrons drops until the losses due to ionization are greater than losses from bremsstrahlung radiation. An electromagnetic shower will also occur if an electron or positron enters the atmosphere, simply alternating which step, bremsstrahlung radiation or pair production, comes first.

\subsubsection{Cosmic-Ray Induced Showers}

There are three possible types of cosmic radiation entering the atmosphere: gamma rays or a cosmic rays (proton, neutrons, electrons, atomic nuclei, and the correspond-

ing antimatter particles), and neutrinos. When a cosmic ray, enters the atmosphere 


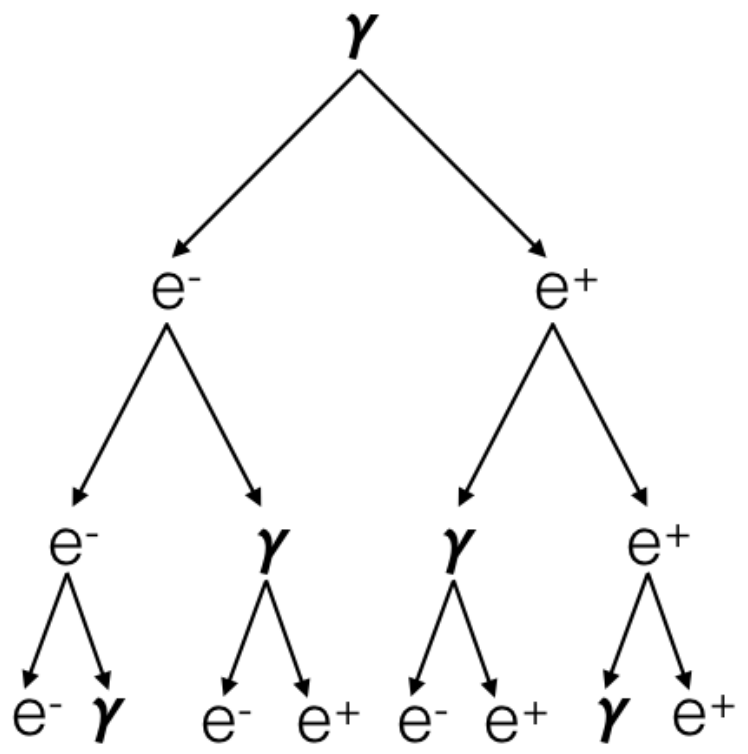

Figure 1.1: Sketch of gamma ray induced air shower a resulting interactions

and collides with nuclei in the atmosphere, pions, other mesons, and baryons are produced. The pions have short life times and decay quickly, with the $\pi^{0}$ decaying into two gamma rays and the $\pi^{ \pm}$decaying into muons and neutrinos [3]. The decay of these pions will produce a cascade of secondary particles shown in Fig. 1.2 [10]. The produced gamma rays will cause a cascade just like that of an originating gamma ray induced shower. The muons though travel further away from the shower core (the path of the original particle) and often reach the earth's surface. Because muons are charged particles and these muons have very high energy they produce Cherenkov radiation. This property of hadronic showers (sometimes referred to as muon-richness) allows detectors to distinguish between gamma-ray showers and hadronic showers. 


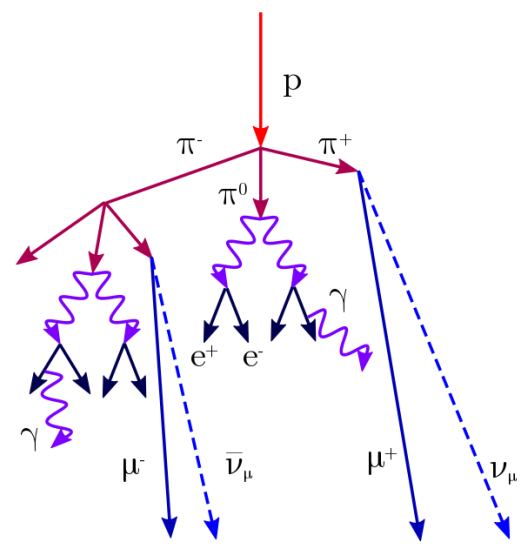

Figure 1.2: Diagram of proton induced shower entering the atmosphere producing initial secondary particles. These showers make up $\sim 90 \%$ of produced air showers [10].

\subsection{Detection with an EAS Array}

\subsubsection{Cherenkov Radiation}

For IACTs and some EAS arrays, like water Chernkov detectors (WCDs), the production of Cherenkov radiation allows for the detection of particles in an EAS. IACTs detect this light in air as the shower develops and WCDs detect this light from air shower particles entering the water tanks of the detector, which will be discussed more in Chapter 2. Cherenkov Radiation is caused by a particle with charge traveling faster than the speed of light in a dielectric medium. This satisfies, 


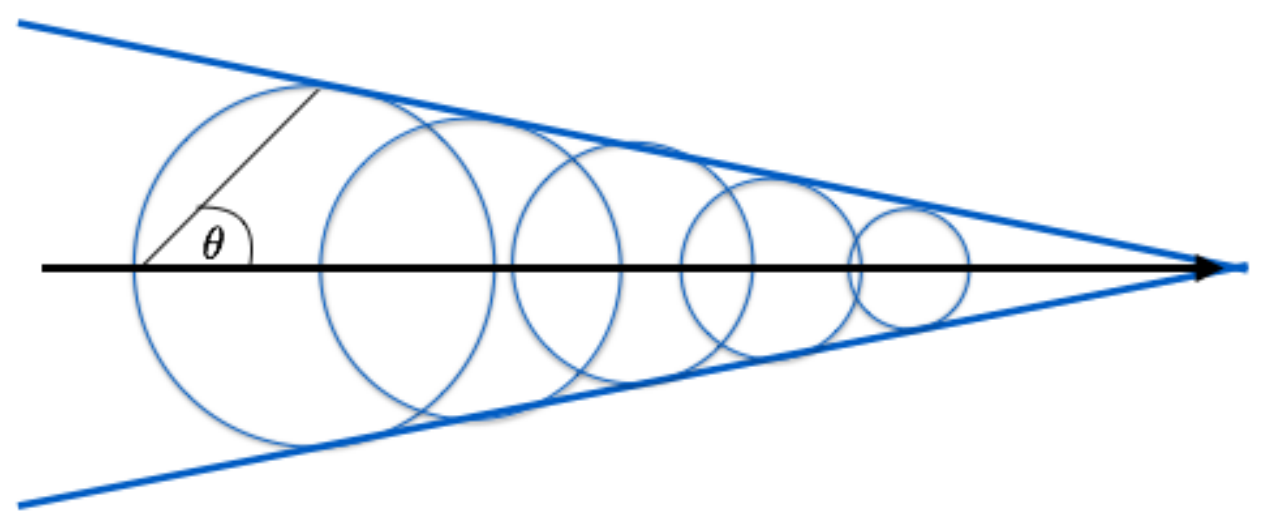

Figure 1.3: Sketch of electromagnetic shock front cause by Cherenkov radiation

$$
\frac{c}{n}<v<c
$$

where $n$ is the index of refraction of the medium. The process creates an electromagnetic shock front, shown in Fig. 1.3, causing the emission of Cherenkov light in a cone with an opening angle given by

$$
\cos (\theta)=\frac{c}{n v_{\text {Particle }}}
$$

The values for the opening angle are $\sim 1.0^{\circ}$ in air and $\sim 40.0^{\circ}$ in water. 


\subsubsection{EAS Arrays}

The atmosphere prevents direct measurement of very high energy cosmic and gamma rays. Since the muons travel straight through the atmosphere, due to being considerably more massive (200 times) than electrons and hence having a larger mean free path, the Cherenkov light remains in a cone of nearly constant angle defined by Eq. 1.3. The presence of the muons and Cherenkov light they create allows for the separation between showers that originate from hadrons and showers from gamma

rays. A simulation shown in Fig. 1.4 shows the difference between a $10 \mathrm{TeV}$ photon and proton induced showers. An EAS array can be used to detect these showers. This is done by using an array of detectors built at high altitude, to be close to the shower maximum. The shower maximum is where energy losses in the shower particles are dominated by ionization losses over bremsstrahlung radiation. There are several different types of detection techniques: using resistive plate chambers measuring the time of particle interaction with them (ARGO-YBJ) [4], using an array of scintillation counters to measure the air shower and underground scintillation counters to measure the muonic component of the shower (KASCADE) [13] and using photomultiplers immersed in water to detect the Cherenkov radiation caused by a particle entering the water [3]. The HAWC observatory described in the next chapter is the latter type of EAS array. 

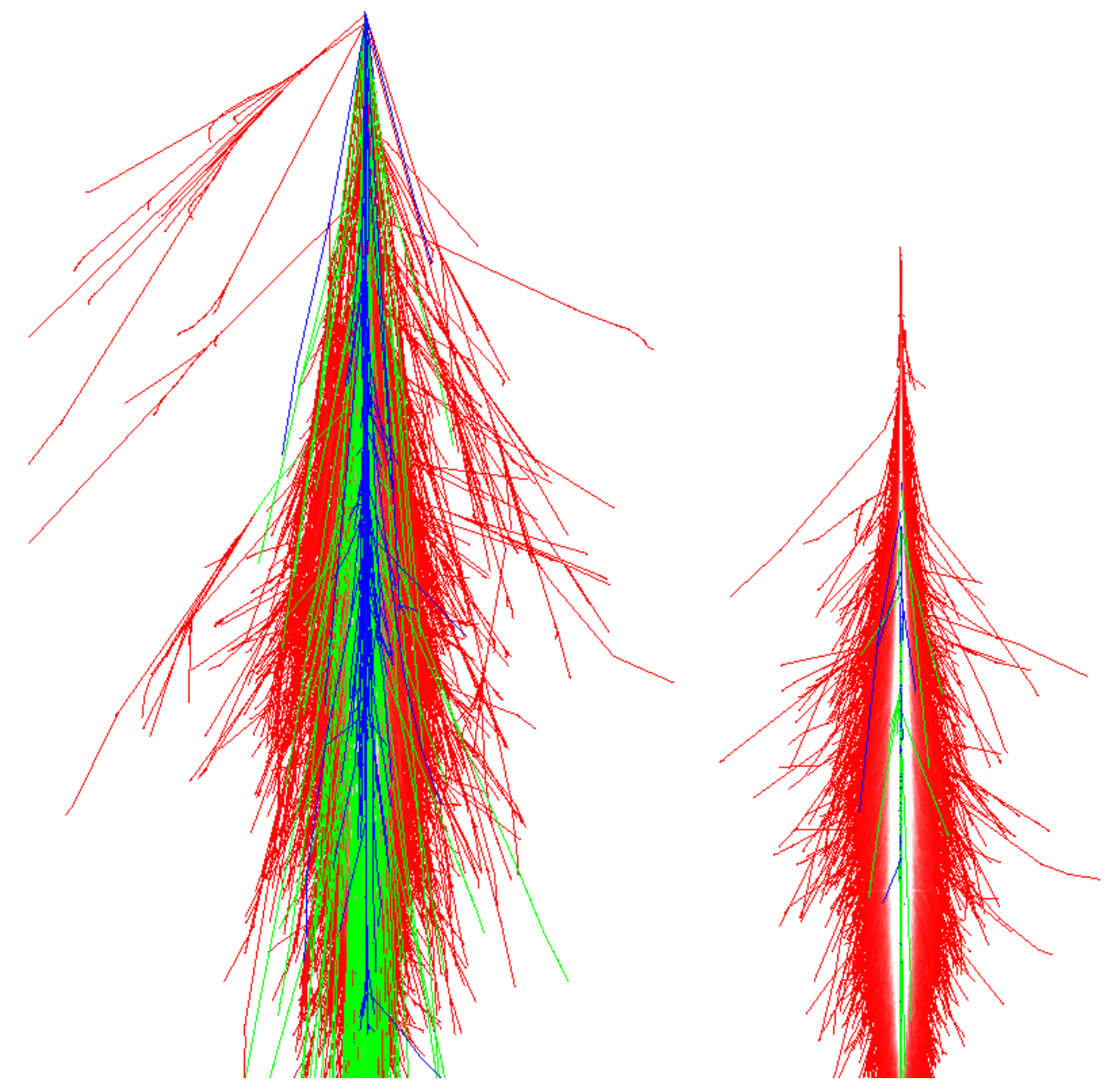

Figure 1.4: Simulations of $10 \mathrm{TeV}$ proton (right) and photon (left) induced showers. Red is the electromagnetic portion (electrons, positrons, photons), blue is the hadronic component, and green is the muon component. CORSIKA software was used to make these plots [7] [3]

\subsection{Multivariate Analysis}

This section is based on information gained through the TMVA User Guide [11]. Using a multivariate analysis for classifying data into two categories has several advantages. First, it allows for separating the phase space more precisely. Second, 
correlations between separation variables are made visible. Third, it takes multiple variables and condenses the information down to a single classification variable that determines the classification of the data by outputting a classification parameter. When classifying between signal and background events, signal events have a larger classification parameter and background events have a smaller classification parameter. There are multiple methods for implementing a multivariate analysis. For the analysis in Chapter 4, boosted decision trees (BDTs) were used and will be described in the following sections.

\subsubsection{Decision Trees}

Decision trees are binary trees that have yes/no criteria that separate a parent node into two child nodes. This is used to separate events into two categories, for instance signal events and background events. This decision is made by taking a separation parameter and applying a cut on it at each level. An example tree is shown in Fig 1.5 . The separation parameter is chosen to increase the separation index, $G$,

$$
G=P(1-P)
$$

between the parent node and the sum of the two child nodes. $P$ is the purity of the signal. The nodes continue to be separated until one of three conditions is met in 


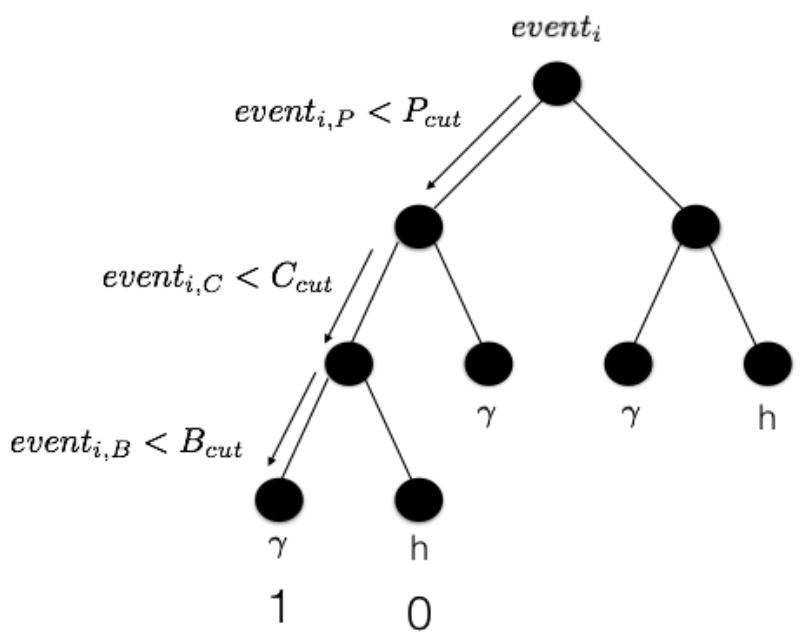

Figure 1.5: Example decision tree with separation parameters being cut on at each level, where $\mathrm{B}, \mathrm{C}$, and $\mathrm{P}$ are properties of the events.

the leaf node: first a maximum number of splits defined by the user has occurred, second a minimum number of events defined by the user are left in the leaf node, third a leaf node contains only one type of event. The leaf nodes are classified as signal-like or background-like based upon the number of resulting events of a given type in that node. The classification of leaf nodes can be done one of two ways. One way is using the majority of events in the leaf node and assigning the leaf node a value of 0 (background) or 1 (signal) based upon whether the events in a node are majority signal or background. Alternatively the node value can be based upon the purity of the leaf node with a value ranging from 0 (background) to 1 (signal). A single decision tree is not good at classifying an event, either because it has learned an example set of data perfectly as each leaf node contains only one event, or because most leaf nodes contain significant impurities. Boosting methods take relatively weak classifiers like decision trees and enhance them, increasing the classification abilities 
of multivariate methods.

\subsubsection{Boosting methods}

The three boosting methods of interest for decision trees are the adaptive boost (AdaBoost), bagging, and randomizing the trees. Each method results in multiple trees that form a forest. The AdaBoost method takes the events that were misclassified in the previous tree and weights them with a singular weight, $\alpha$, given by

$$
\alpha=\frac{1-e r r}{e r r}
$$

where $\operatorname{err}$ is the rate of events misidentified in the previous tree. After the weighting is applied, the events in the sample are renormalized so that the sum of the weights is constant. The classification parameter, $h_{i}(x)$ for individual decision trees can be defined in one of two ways. First is \pm 1 where +1 is a signal like event and -1 is a background event. Alternatively it can be defined as the purity, $P$, of the node given by

$$
h_{i}(x)=P=\frac{S}{S+B}
$$


where $S$ is the number of signal events and $B$ is the number of background events in the end node. The response value from the forest, $y_{b o o s t}$, can then be defined by

$$
\left.y_{\text {boost }}=\frac{1}{N_{\text {collection }}} \sum_{i}^{N_{\text {collection }}} \ln \left(\alpha_{i}\right) h_{i} \dot{(} x\right)
$$

where $N_{\text {collection }}$ is the number of trees produced, and $x$ is the tuple of separation parameters.

Bagging is the process of resampling the training sample for each tree with the replacement. This allows for the selection of an event more than once when creating each tree, thus causing the entire training sample to function as a probability density function of the phase space. As each individual tree uses a different subsample of events the classifiers are independent of one another and can be combined into a collection, and the classifiers can be averaged to get a response value that is stronger than each tree would be individually.

Randomizing trees changes the way the splitting parameter is chosen. Each time a tree is split, instead of selecting the variable that maximizes the separation gain at each split, only a subsample of the separation variables is examined and a variable from this subsample is chosen that maximizes the separation index. This method requires that bagging is used when constructing the forest of trees. All three of these methods are used together in the Chapter 4 analysis. 
This thesis is organized as follows. Chapter 2 will discuss the HAWC observatory, and the air shower reconstruction HAWC uses. Chapter 3 covers the calibration procedure. Chapter 4 describes the multivariate analysis with the TMVA software package, on the Crab Nebula. Conclusions from the presented work and an outlook on future are provided in Chapter 5. 



\section{Chapter 2}

\section{HAWC Observatory}

\subsection{Detector}

The High Altitude Water Cherenkov (HAWC) observatory is fully operational since March 2015, with 300 Water Cerenkov Detectors (WCD) which are cylindrical steel tanks $7.3 \mathrm{~m}$ in diameter and $5 \mathrm{~m}$ tall. Each tank contains four photomultipler tubes (PMTs) submerged in $\sim 200,000$ liters of purified water. The observatory is located on the Sierra Negra plateau at an altitude of $4100 \mathrm{~m}$. An aerial view of the detector is shown in Fig. 2.1. The detector covers an area of $22,000 \mathrm{~m}^{2}$ and the effective area varies depending upon the energy, increasing with energy [1]. The PMTs in each tank consist of three 8-inch Hamamatsu R5912 PMTs [8] placed at a radius of $1.8 \mathrm{~m}$ from 


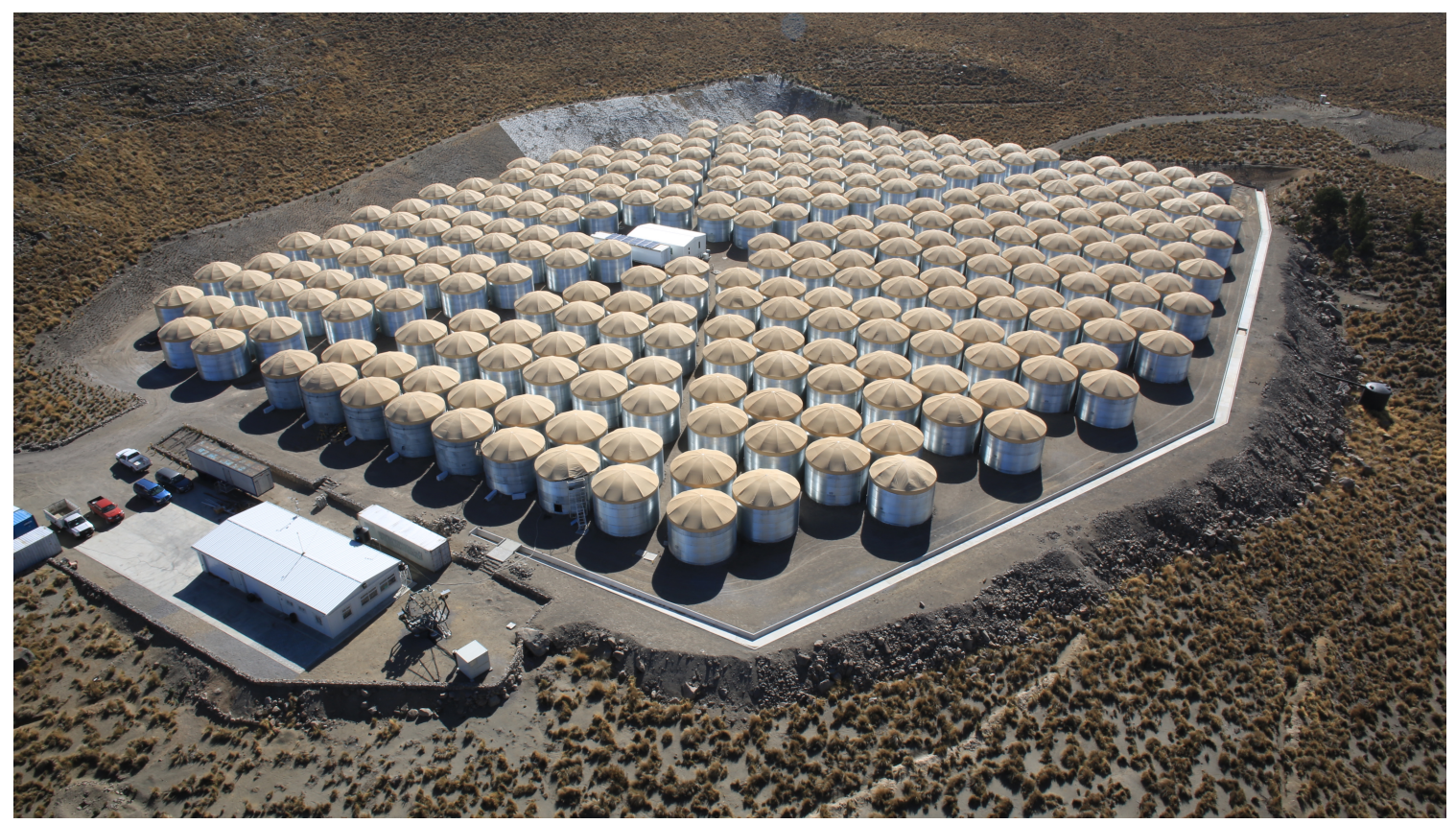

Figure 2.1: The HAWC Detector Array.

the center and one 10-inch Hamamatsu R7081 PMT [9] placed at the center of the tank, shown in Figure 2.2 [10] [22]. The HAWC observatory is sensitive to cosmic rays with energies between $100 \mathrm{GeV}$ and $100 \mathrm{TeV}$, and can view two thirds of the sky, with an instantaneous field of view of 2 sr [3].

\subsection{Event Reconstruction}

An air shower event can be detected by the array of PMTs as the shower front crosses the detector. Whether an event is detected or not depends upon the size of the air shower, the energy of the shower, and whether it hits a minimum of $6.7 \%$ of properly calibrated PMTs. Photons from the Cherenkov radiation of the electrons entering 


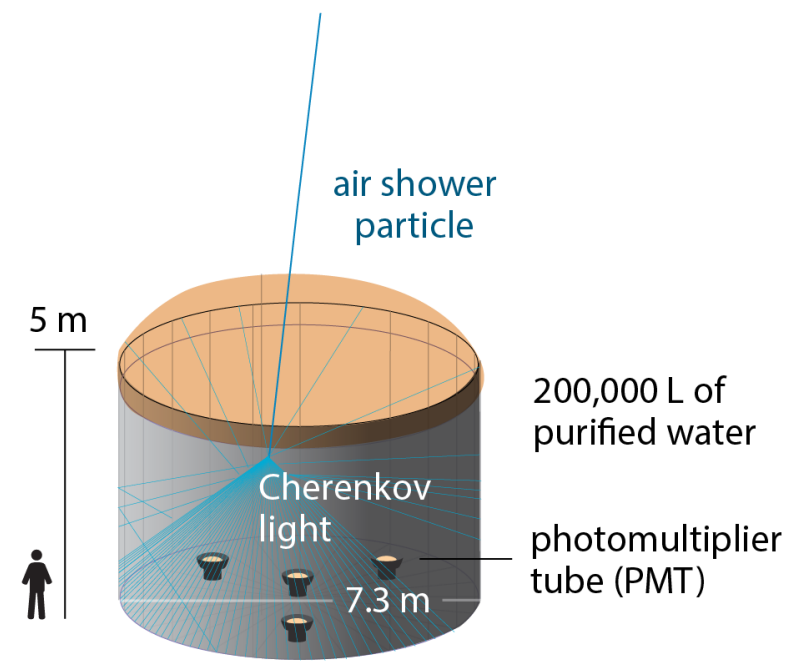

Figure 2.2: Tank schematic, showing PMT setup with optical fibers and diffuser [10].

each tank that hit a PMT within a certain time window are grouped together as one event. The process of reconstructing an event is broken down into three parts: edge finding, the core fit, and the angular fit.

\subsubsection{Edge Finding}

An event is comprised of hits, which are when a PMT detects Cherenkov light produced by a high energy charged particle entering the water in a tank. A hit includes the position (given by the PMT location), the charge, and the time of the hit. The charge and time of the hit come from the data acquisition system (DAQ) once it has been calibrated by the laser system. The DAQ has two voltage thresholds in units of photoelectrons (PEs) that are used to determine if a PMT is hit and it records 
the times that the voltage thresholds are crossed. An example of the voltage pulse from a hit PMT is shown in Fig. 2.3. The time it takes for the same threshold to be crossed twice is called time over threshold (ToT). There are two thresholds levels. The low ToT is the amount of time the magnitude of a pulse is larger than the low threshold, corresponding to 0.25 photo electrons (PE). The high ToT is the amount of time the magnitude of a pulse is larger than the high threshold corresponding to 4.0 PEs [3]. Each time a pulse crosses a threshold creates an edge, as shown at the bottom of Fig. 2.3. Events with signal $>4.0$ PE, that cross both thresholds, are called 4-edge hits, and events with signal between 0.25 PE and 4.0 PE, crossing only the low threshold, are called 2-edge hits. As the only information recorded is the time of threshold crossing, not the direction of crossing or which threshold is crossed, it is possible to misidentify two 2-edge hits as one 4-edge hit. To determine if the hit is a 2-edge or 4-edge hit the time between the $t_{0}$ and $t_{1}\left(T_{01}\right)$ is cut on. The hardware ensures that low ToT is longer than 53 ns. Because the rise time of a 4-edge hit is expected to be less than $53 \mathrm{~ns}$, this is used for cut value of $T_{01}$ to determine if a pulse is a 2-edge event or a 4-edge event [24].

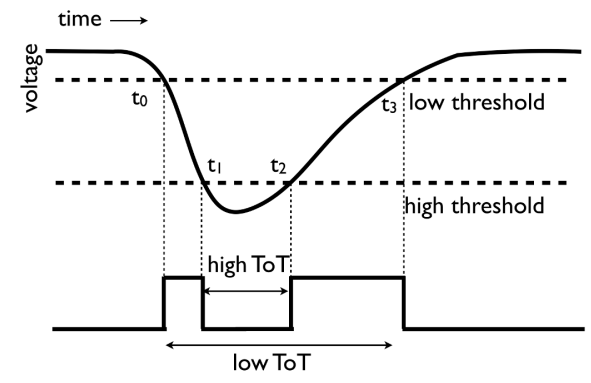

Figure 2.3: High and Low threshold sample of signal (voltage) pulse and the edges produced by threshold crossing [24]. 


\subsubsection{Core Fit}

Two algorithms are used to find the shower core. The first uses a simple center-ofmass procedure, that estimates the initial core position which is defined by

$$
\begin{aligned}
x_{\text {com }} & =\frac{\sum x_{i} q_{i}}{\sum q_{i}} \\
y_{\text {com }} & =\frac{\sum y_{i} q_{i}}{\sum q_{i}}
\end{aligned}
$$

where $q_{i}$ is the charge of a hit within the event and $x_{i}$ and $y_{i}$ are the $\mathrm{x}$ and y position of the PMT hit. This position is an initial guess for the second algorithm [24].

The second algorithm is a $\chi^{2}$ minimization,

$$
\chi^{2}=\sum \frac{\left(q_{i}-Q_{i}\right)^{2}}{\delta q_{i}^{2}}
$$

that compares the measured charge $q_{i}$ to the expected charge $Q_{i}$ where $\delta q_{i}$ is the the error in the measured charge. $Q_{i}$ has been defined by various charge distributions. Initially the charge distribution was modeled by a Gaussian function defined by

$$
Q\left(x_{i}, y_{i}, N-x_{0}, y_{0}, \sigma\right)=\frac{N}{2 \pi \sigma^{2}} \exp \left(-\frac{1}{2}\left(\left(\frac{x_{i}-x_{0}}{\sigma}\right)^{2}+\left(\frac{y_{i}-y_{0}}{\sigma}\right)^{2}\right)\right)
$$

where $N$ is the amplitude of the shower, $\sigma$ is the width of the shower core, and 
$x_{0}, y_{0}$ are the coordinates of the core position [3]. A better estimation of the charge distribution for electromagnetic air showers is the Nishimura-Kamata-Greisen (NKG) function defined by

$$
Q\left(x_{i}, y_{i}, N-x_{0}, y_{0}, s\right)=\frac{N}{2 \pi R_{M o i}^{2}} \frac{\Gamma(4.5-s)}{\Gamma(s) \Gamma(4.5-2 s)}\left(\frac{r}{R_{M o l}}\right)^{s-2}\left(1+\frac{r}{R_{M o l}}\right)^{s-4.5}
$$

where $N$ is the total number of electrons in the shower event, $s$ is the shower age, which parametrizes the stage of shower development. When $s=1$ the number of particles in the shower plane is at a maximum [3]. $R_{M o l}$ is defined by

$$
R_{M o l}=X_{0} \frac{E_{s}}{E_{c} \rho}=\left(37.15 \mathrm{gcm}^{-2}\right) \frac{21 \mathrm{MeV}}{(84.4 \mathrm{MeV})\left(7.4 \times 10^{-4} \mathrm{gcm}^{-3}\right)}=124.21 \mathrm{~m}
$$

The constants $X_{0}, \rho, E_{s}$, and $E_{c}$ are the radiation length, air density at observation height, the scattering energy and critical energy where energy losses from bremsstrahlung and ionization are equal, respectively [3]. The current distribution is a compromise between a Gaussian function and the Nishimura-Kamata-Greisen (NKG) function called the Gaussian-NKG function. The NKG function is more accurate but computationally expensive than the Gaussian-NKG. The Gaussian-NKG 


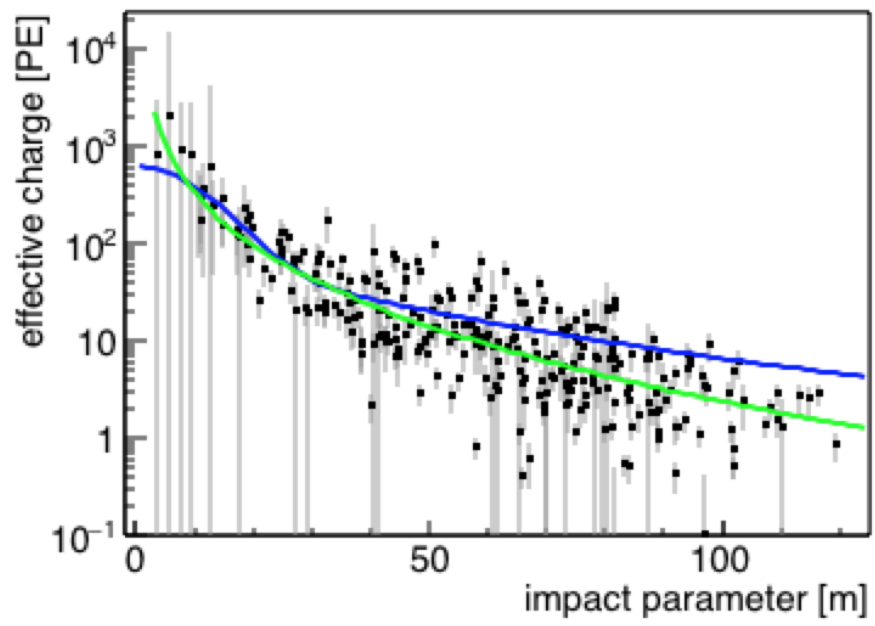

Figure 2.4: Charge distribution as a function of the distance of the PMT location (location of the charge measurement) from the core. Showing best fit of gaussian-NKG function after the $\chi^{2}$ minimization (Blue) and the NKG fit using the core position from the gaussian-NKG function (Green) needed for determining the shower age and amplitude parameters [3].

function is defined by

$$
Q\left(x_{i}, y_{i}, N, x_{o}, y_{o}\right)=A\left(\frac{1}{2 \pi \sigma^{2}} e^{\frac{-x^{2}}{2 \sigma^{2}}}+\frac{N}{\left(0.5+\frac{r}{R_{M o l}}\right)^{3}}\right)
$$

where $r$ is the distance between shower core and PMT position, $N$ is the normalization of the integral of the second term relative to the Gaussian, and is equal to $5 \times 10^{-5}$, $\sigma$ is the width of the shower core and $x_{o}$ and $y_{o}$ define the core position, $A$ is the amplitude of the distribution, and $R_{M o l}$ is defined by Eq. 2.6. The core distributions of some EAS for the Gaussian-NKG function are shown in Fig. 2.4 [3]. 


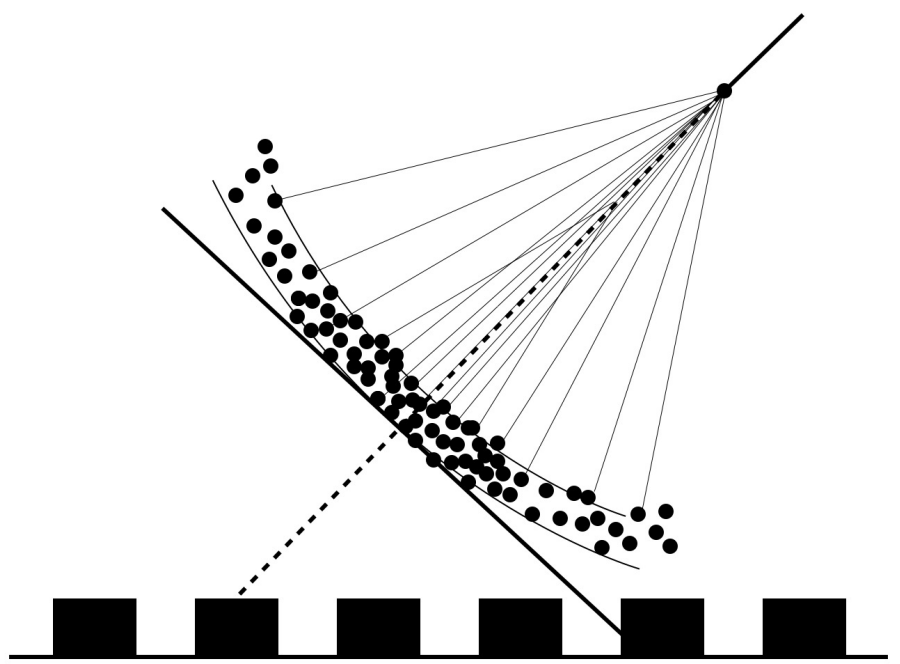

Figure 2.5: Example shower front hitting detector [24].

\subsubsection{Angular Fit}

The angular fit determines the direction of the primary particles that causes the EAS. The shower front is curved as shown in Fig. 2.5. A curvature correction is applied and corresponds to a projection of the shower front onto a plane. The correction depends on the lateral distance of a detected signal from the shower core. The curvature correction is approximately $0.07 \mathrm{~ns} / \mathrm{m}$. An additional correction, the sampling correction accounts for the thickness of the shower front which also varies with the lateral distance from the core. This correction is needed because there are fewer secondary particles further from the core which delays the detection of the corresponding (smaller) PE signal in PMTs further from the shower core. Both corrections are obtained using a large number of simulations of EAS [24]. 
Table 2.1

$f_{\text {hit }}$ bins where $f_{\text {hit }}$ is the percentage of properly calibrated PMTs participating in an event [1].

\begin{tabular}{cc}
$\beta$ & $f_{\text {hit }}$ \\
\hline 1 & $6.7-10.5 \%$ \\
2 & $10.5-16.2 \%$ \\
3 & $16.2-24.7 \%$ \\
4 & $24.7-35.6 \%$ \\
5 & $35.6-48.5 \%$ \\
6 & $48.5-61.8 \%$ \\
7 & $61.8-74.0 \%$ \\
8 & $74.0-84.0 \%$ \\
9 & $84.0-100.0 \%$
\end{tabular}

\subsection{Gamma/Hadron Separation}

Separating the gamma rays (signal) from the hadrons (background) is important. As mentioned gamma rays retain source information as they are not deflected by interstellar magnetic fields. Air shower events are separated into bins based upon $f_{\text {hit }}$, the number of properly calibrated and active PMTs hit by the air shower, as the ability to separate signal from background is dependent upon shower size. The $f_{\text {hit }}$ bins are defined in Table 2.1] [1]. The shower age and the shower amplitude, which are derived from the NKG function Eq. 2.5 are used in addition to the parameters described in the following section in the analysis in Chapter 4. 


\subsubsection{PINCness and Compactness}

The two current parameters for separation between gamma rays and hadrons: the parameter for identifying nuclear cosmic rays (PINCness), and Compactness. Compactness is a measure of how tightly grouped the charge distribution is around the core and is defined as,

$$
C=\frac{N_{h i t}}{C x P E_{40}}
$$

where $N_{\text {hit }}$ is the number of PMTs participating in the event and $C x P E_{40}$ is the largest measured charge outside of a $40 \mathrm{~m}$ circle of the shower core. This is used as muons are expected to travel far from the shower core and produce higher measured charge, and the presence of high energy muons indicate that it is more likely to be a hadronic shower. PINCness is a measure of how smooth the charge distribution is and is defined by,

$$
P=\frac{1}{N} \sum \frac{\left(\log \left(q_{i}\right)-<\log \left(q_{i}\right)>\right)^{2}}{\left(\sigma_{\log \left(q_{i}\right)}\right)^{2}}
$$

where $\log \left(q_{i}\right)$ is the log of the measured charge in a PMT, $<\log \left(q_{i}\right)>$ is the average charge in all PMTs inside of an annulus with a width of $5 \mathrm{~m}$ centered on the core, and containing charge, $q_{i}$, and $\sigma$ is the uncertainty in the charge [3]. This detects 
fluctuations within in a radial distance from the core, in gamma ray showers the fluctuations should be small as gamma-ray showers tend to be symmetric where as hadronic showers do not. In Fig. 2.6 are the PINCness vs. Compactness distributions for gamma rays and hadrons for $f_{\text {hit }}$ bin 9 .

\subsection{Calibration System}

The purpose of the calibration system is to calibrate the DAQ. The calibration system is used to measure the response time of the two threshold TDC DAQ, and it converts the ToT to charge values in $\mathrm{PE}$. The calibration system contains a laser connecting to a series of filter wheels, fiber optic cables, optical splitters and diffusers going to each WCD. The $532 \mathrm{~nm}$ laser is pulsed using a square wave generator [18]. This laser was chosen for several reasons: a compromise between optic-fiber transmission efficiency and quantum efficiency of the PMTs, the laser pulse width needed to be smaller than the shower front width and the pulses needed to produce photons of $45 \mu \mathrm{J}[14]$. This light pulse travels along paths shown in Fig. 2.7 [14]. After passing through the first splitter, a subbeam is directed to a radiometer, RAD1, to monitor the laser power. The remaining beams go through a series of three filter wheels each with six neutral density filters of different optical depths [20], allowing for laser intensities over six orders of magnitude of intensity. Only 68 different combinations of filter wheel settings are used, as some combinations result in the same optical depth. This results 


\section{Gamma Smoothness Histogram}
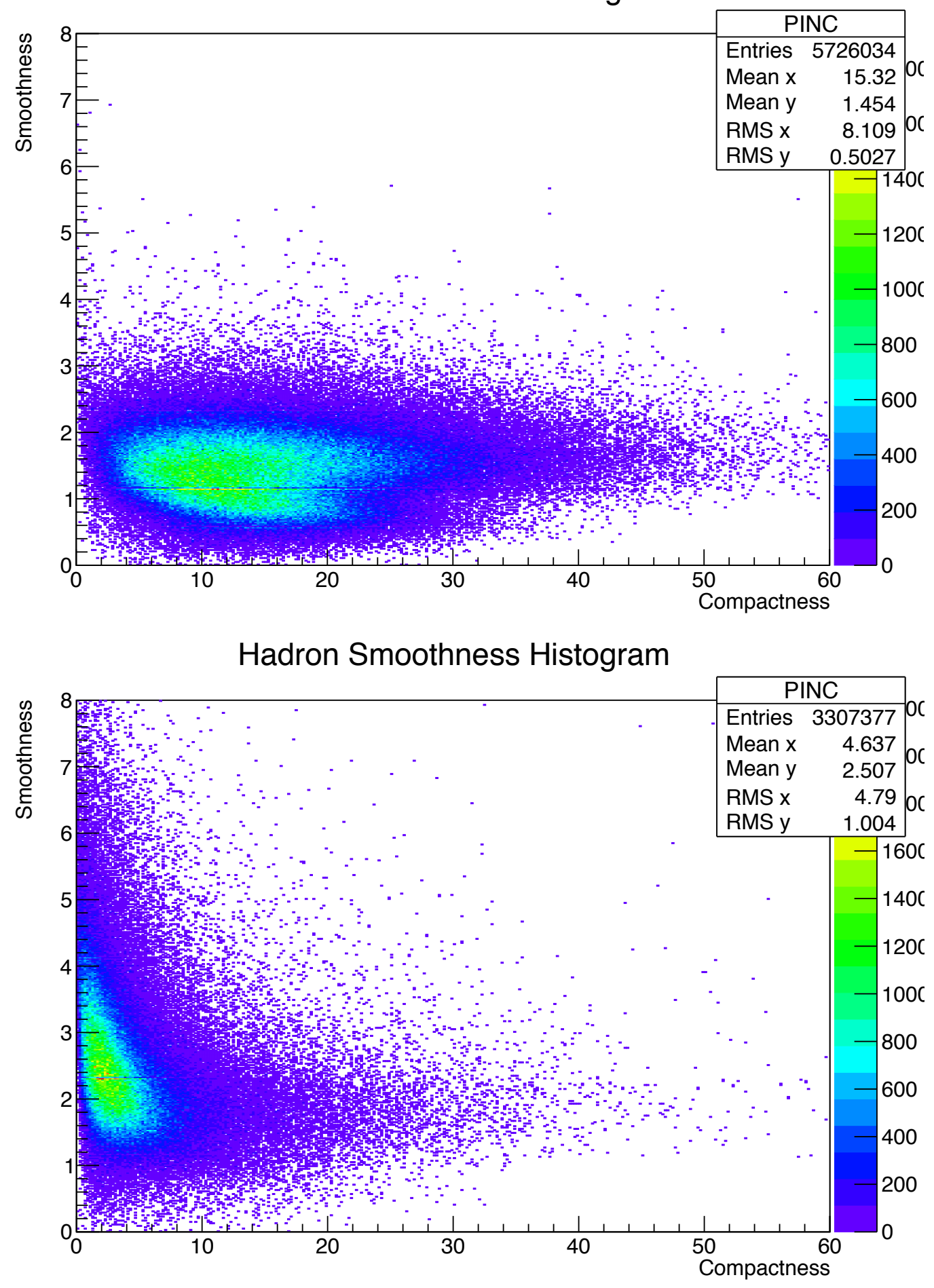

Figure 2.6: The gamma ray (top) and hadron (bottom) distributions of PINCness vs. Compactness for $f_{\text {hit }}$ bin 9 . 
in optical depths, $\alpha$, as defined by

$$
\text { Transmittance }=10^{\alpha / 10}
$$

from 0.0 to 6.5 [23]. For each filter wheel combination 2000 laser pulses are sent. The pulse is then directed through a series of fiber optic cables and optical splitters. To correct for this travel time the total optical travel time for each PMT was measured by inserting a Luna optical backscatter reflector after the splitters and measuring the optical travel time in both directions [15] [17. This pulse travel is then accounted for in the slewing calibration described in Chapter 3. As shown in the calibration setup the $T_{\text {start }}$ and $T_{\text {stop }}$ times are recorded by the DAQ, these times being the start and stop times for a laser pulse going all the way through the calibration system. Signals that have a $T_{\text {stop }}$ within $2 \mu \mathrm{s}$ of the start time are then used for the calibration. 24] 


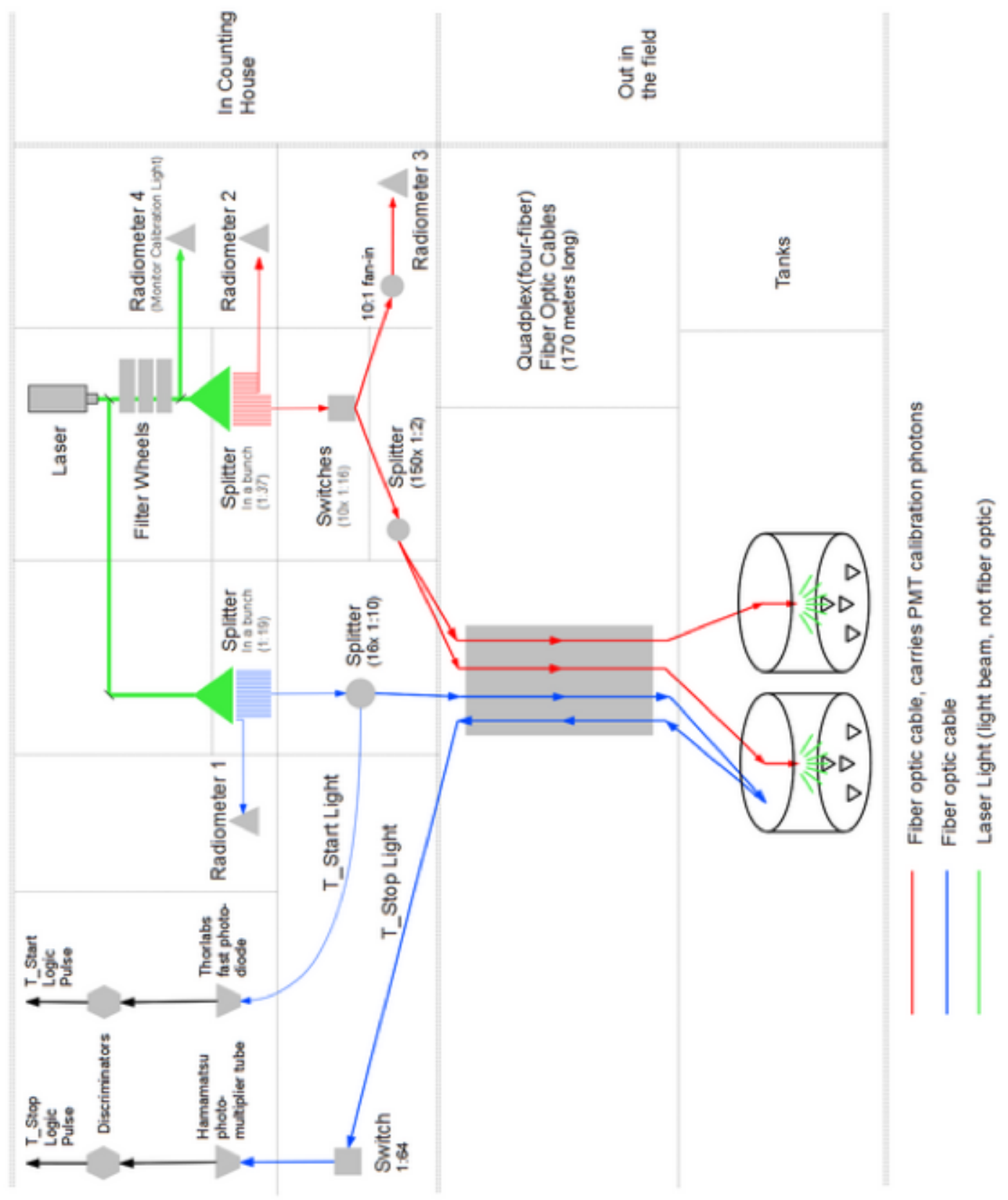

Figure 2.7: Layout of HAWC calibration system [14]. 


\section{Chapter 3}

\section{Calibration Procedure}

\subsection{Calibration Steps}

The calibration of the detector is divided into several parts: the charge calibration, the timing calibration, the zenith alignment, and the bad channel determination. The purpose of the charge calibration is to convert the ToT recorded by the DAQ (described in Chapter 2) into charge in units of PE. The purpose of the timing calibration is to measure individual PMT response time. The zenith alignment corrects for a potential offset in the global pointing of the detector, based on the measured zenith angle distribution of detected EAS events. The bad/good channel determination provides a list of channels that pass a set of data and calibration quality requirements 
and that can be used when reconstructing data for a given time period. It consists of a charge autocheck, a slewing autocheck, a time residual autocheck, and a set of diagnostic checks.

\subsection{Charge Calibration}

When an event crosses the plane of the detector it is recorded if the event triggers at least $6.7 \%$ of active PMTs. A PMT is triggered if the electrical pulse crosses at least one of the two voltage thresholds of the DAQ. The time over threshold, ToT, is the time between the leading and trailing edges of the electrical pulse. Because ToT is directly related to the amplitude of the electrical pulse, it can be converted to an amount of charge in the pulse. The ultimate goal of the charge calibration is to convert ToT to the number of photon electrons, PEs, in a pulse [21]. The charge calibration consists of two parts. The first is the occupancy method which determines the relationship between the number of PEs for a given ToT for each PMT. The second is an automatic check that examines the distributions found using the occupancy method to determine if a channel is properly calibrated. 


\subsubsection{Occupancy method}

To determine the response of each individual PMT, an occupancy method is used to determine the number of PEs detected. It is assumed that the number of PEs detected by a PMT $\left(N_{P E}\right)$ follows a Poisson distribution. The occupancy, $\eta$, is defined by

$$
\eta=\frac{N_{\text {laser }}}{N_{\text {trigger }}}=1-e^{-<N_{P E}>}
$$

where $N_{\text {laser }}$ is the number of laser pulses whose signal size exceeds the threshold of a given PMT and $N_{\text {trigger }}$ is the total number of laser pulses sent from the calibration system. The occupancy is also equal to the probability of seeing at least one PE [24]. The mean number of PEs, $\left\langle N_{P E}\right\rangle$, can be defined by

$$
<N_{P E}>=-\ln (1-\eta)
$$

For higher intensities this does not work as the error of $\left\langle N_{P E}\right\rangle$ increases as the occupancy goes to one, but it can be assumed that $\left\langle N_{P E}>\right.$ is linearly related to the laser intensity above $2 \mathrm{PE}$. Setting $\left\langle N_{P E}>\right.$ found from Eq. 3.2 equal to the following 


$$
<N_{P E}>=A\left(\frac{I}{I_{r e f}}\right)+B
$$

where $I$ is the intesity measured by the radiometer, $I_{r e f}$, which is $10^{-8} \mathrm{~J}$, is the maximum intensity used in the calibration run, and $B$ is a constant offset $(\sim 0)$. This allows for $A$, the maximum number of PEs the PMT can accurately measure, to be solved for [21]. From this with a simulated distribution of the actual PEs using a Poisson distribution with a mean of $\left\langle N_{P E}\right\rangle$, a conversion equation can be determined, where the $\log \left(N_{P E}\right)$ is a function of ToT. In Fig. 3.1 21] the charge vs ToT distribution is shown and fitted with a 6 parameter broken power law, defined by

$$
\log \left(N_{p e}\right)= \begin{cases}p_{1}+p_{2} \times T o T & p_{\min }<T o T<p_{4} \\ \left(p_{1}+p_{2} \times T o T\right) \times\left(e^{\frac{T o T-p_{4}}{p_{3}}}-\frac{T o T-p_{4}}{p_{3}}\right) & p_{4}<T o T<p_{\max }\end{cases}
$$

where $p_{1}$ and $p_{2}$ describe the linear portion at low values of ToT. $p_{3}$ and $p_{4}$ describe the exponential portion of the data with $p_{4}$ also defining the point where the linear fit alone no longer well describes the data. $p_{\min }$ and $p_{\max }$ are the lower and upper limits of ToT for which the charge calibration is valid. 


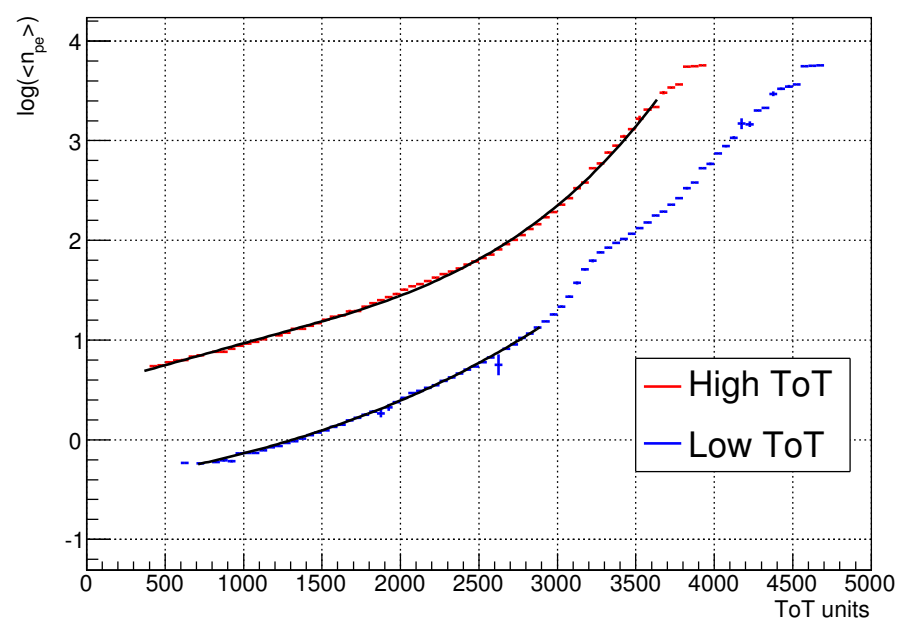

Figure 3.1: Plot of $\log \left(N_{P E}\right)$ as a function of ToT counts for one PMT channel in the HAWC array [21].

\subsubsection{Charge Calibration Autocheck}

After the charge calibration is completed an automated check is performed. This looks at the charge distribution of PMTs resulting from using the calibration to convert the ToT of trigger data to PE values. In Fig. 3.2 is the distribution for one PMT. A single photo-electron (SPE) peak can be seen in the distribution. The physical reason for this peak is there is a high probability of a air shower particle generating only one $\mathrm{PE}$ [21]. For this check the PE distribution at low charge values is fit with a double Gaussian,

$$
f(x)=p_{0} e^{-\frac{\left(x-p_{1}\right)^{2}}{2\left(p_{2}\right)^{2}}}+p_{3} e^{-\frac{\left(x-2 p_{1}\right)^{2}}{4\left(p_{2}\right)^{2}}}
$$




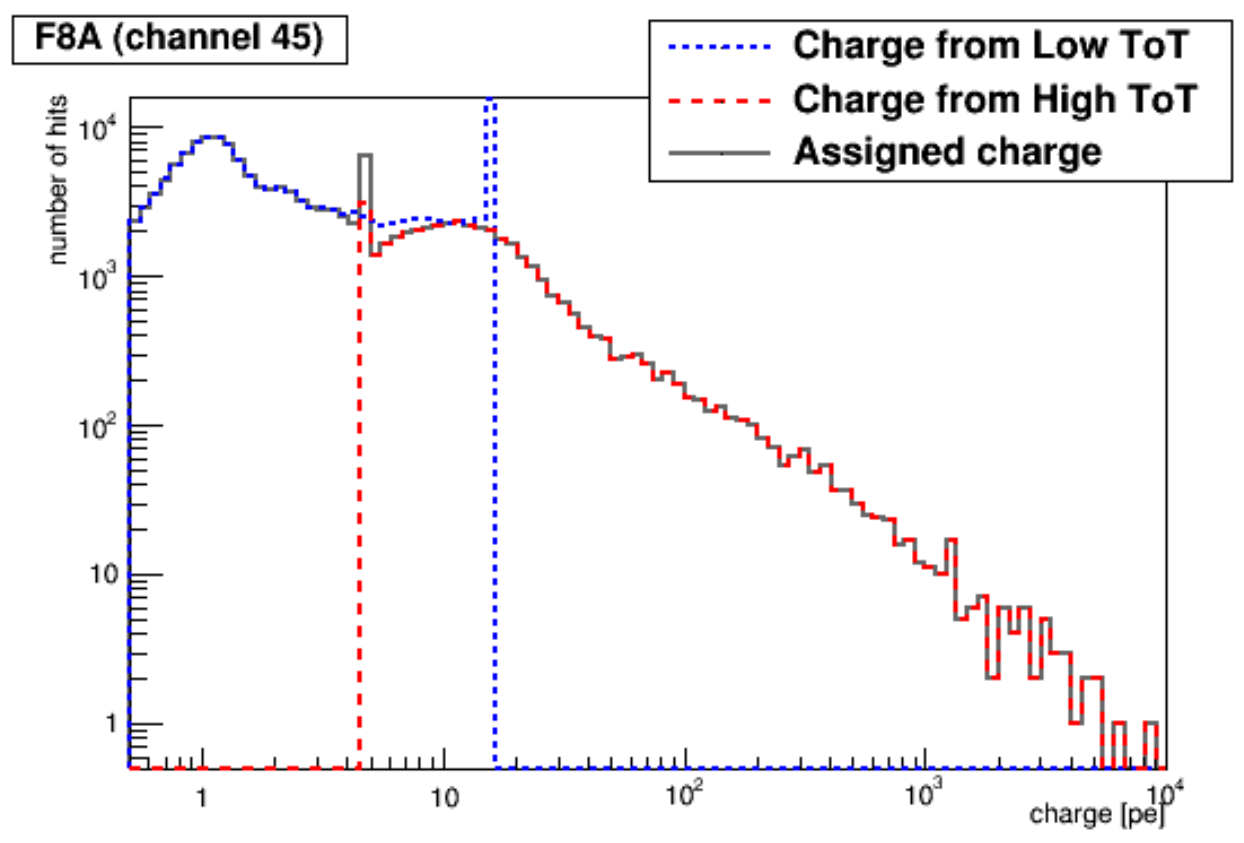

Figure 3.2: Charge distribution of PMT F8A [21].

where $p_{0}$ and $p_{3}$ are normilzation factors for each Gaussian, $p_{1}$ is the SPE value, and $p 2$ is the width of the SPE gaussian (sigma). The distributions of the SPE value and the SPE width are shown in Fig. 3.3 [21]. PMTs that had SPE values or SPE widths more than four standard deviations from the mean were declared bad. This list of bad channels is then added to the other lists of channels determined by the slewing, timing and diagnostic checks described in the following sections.

\subsection{Timing Calibration}

The timing calibration is divided into two parts: the individual PMT response time based upon the ToT of a PMT pulse, hereafter referred to as the slewing time, and 

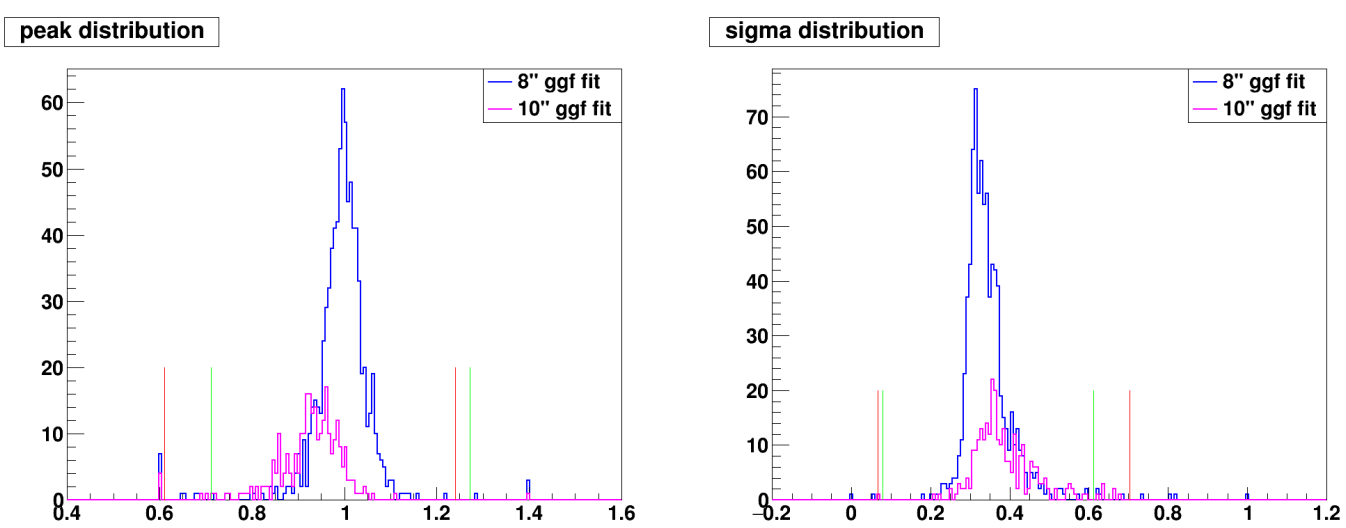

Figure 3.3: Distributions of the SPE value (left) and the width of the SPE gaussian (right) for 8"(blue) and 10" (magenta) PMTs. The cut value lines for the 8" PMTs (red) and 10" PMTs (green) are also shown [21].

the relative timing between PMTs across the HAWC array. To determine the slewing time, the laser system described in Chapter 2 is used to simulate PMT responses across the ToT distribution that correspond to the PE range for air shower particles that are detected by the HAWC Observatory. The relative timing between PMTs is determined by reconstructing data and corrected by comparing the actual PMT timing and the timing expected from the EAS event reconstruction.

\subsubsection{Slewing Calibration}

The slewing calibration refers to the determination of PMT response time. The rise time of an electronic signal is measured using a short, 300 ps laser pulse. The rise time depends upon the intensity of the incoming light and the rise time decreases with increasing intensity. The raw slewing time is equal to the difference between the 
initial laser firing time, $T_{\text {start }}$, and the time when the low threshold is crossed. This time includes not only the individual response time of each PMT but also the travel time along the optical fibers, and splitters, and through the diffuser and the water in the WCD. The travel time is subtracted from the raw slewing time to calculate the actual rise time, $\Delta t_{\text {start }}$. This subtracted time includes the individually measured optical paths and the WCD geometry a 2.2 ns offset between the central PMT and the outer PMTs shown in Fig. 2.2. The slewing time for each PMT, $\Delta t_{\text {start }}$, is plotted vs ToT, where ToT is binned for both low and high threshold, in bins of 9.8 ns width. Only bins with at least 200 entries are used, to ensure sufficient statistics are available to perform a Gaussian fit to the $\delta t_{\text {start }}$ distribution in each ToT bin. Fig. 3.4 shows the mean value resulting from the best fit plotted vs the respective ToT for the four PMTs in WCD G18. A fitting function,

$$
\operatorname{Slewing}(T o T)=e^{\frac{-T o T-p_{0}}{p_{1}}}+p_{2}-p_{3} * T o T
$$

is iteratively fit to the slewing curve resulting from this procedure. The fitting parameters $p_{0}$ and $p_{1}$ are initially chosen such that the exponential term is approximately one. This allows for the linear portion to be fitted between 150 ns and 300 ns. Then, using the values for the linear portion as initial values for $p_{2}$ and $p_{3}$ the entire equation is fitted to the curve. As expected, the central PMT, represented by the green curve in Fig 3.4, exhibits a different timing. 


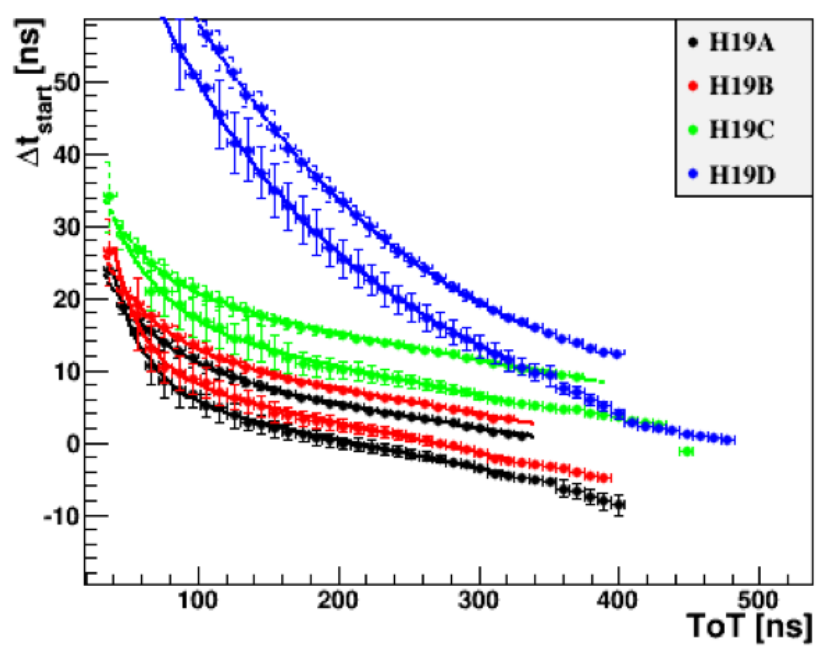

Figure 3.4: Slewing curves of WCD H19, with fitting function. The two populations for each PMT are for the high and low threshold with the high threshold fit being above the low threshold fit. The A,B and D PMTs are 8" PMTs. The C PMT is the 10" PMT and installed at the center of the WCD

\subsubsection{Slewing Autocheck}

The slewing autocheck was originally produced so that an individual would not have to determine the quality of the timing calibration in each of the 1200 PMT channels by eye alone. The primary criterion used in determining the quality of the timing calibration is the shape of the fitting function. The check was designed to be applied to previous calibrations without reprocessing the calibration runs. The information available to compare curves was limited to fit parameters since the individual data points used for fitting were not saved. The distributions of the fitting parameters were examined along with a modified $\chi^{2}$ distribution. Ultimately the modified $\chi^{2}$ distribution was used. A set of channels that were identified by eye as bad for each 
previous calibration run were used as a test sample to confirm that the automated check was working.

\subsubsection{Distributions of Fit Parameters}

There are four fit parameters for each PMT, given by Eq.3.6. To identify bad channels

a list of known bad channels from a sub-sample of calibration runs was created as the test sample of bad channels. The distributions for each parameter were examined and the parameter values for the bad channels were compared with the values of the good channels for each parameter. The distributions of the parameters for the low and high threshold curves are in Fig. 3.5 and Fig. 3.6 respectively. The bad PMTs did not have distinct parameter values that fell outside of the typical distribution for each parameter, thus a direct cut on a parameter could not be done, which led to the the development of the following procedure.

\subsubsection{Modifid $\chi^{2}$ Distribution Check}

The modifed $\chi^{2}$ distribution check examines the shape of the slewing fit for each 8inch and 10-inch PMT and compares the shape of the individual fits to the shape of the average slewing fit for 8-inch and 10-inch PMTs from the pervious calibration run. The assumption being that each PMT should behave similarly within some tolerance. 

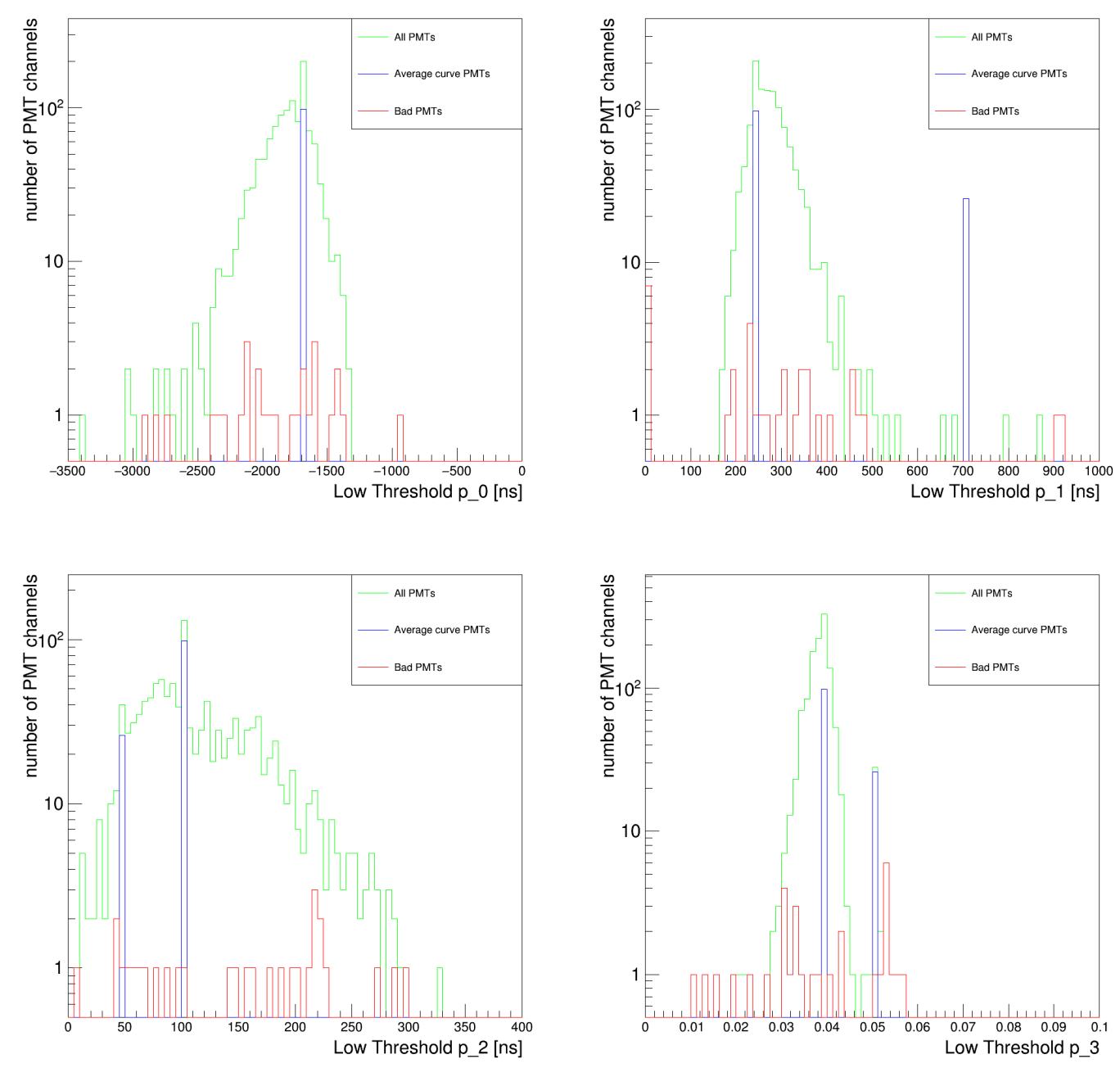

Figure 3.5: The distribution of the slewing fit parameters, $p_{0}$ (upper left), $p_{1}$ (upper right), $p_{2}$ (lower left), $p_{3}$ (lower right) for the low threshold fit. The green distribution contains all 1200 PMT IDs. The blue distribution contains all PMTs assigned the average curve, due to not being active at the time of calibration or not receiving laser light. The red distribution represents the channels known to be bad.

An initial set of PMTs known to be properly calibrated by visual inspection is taken and used to produce an average fit. The average fit is produced by averaging the data points in each ToT bin for all 8-inch and all 10-inch PMTs and then fitting the averaged points with Eq. 3.6. For each calibration run after the first, the average fit 

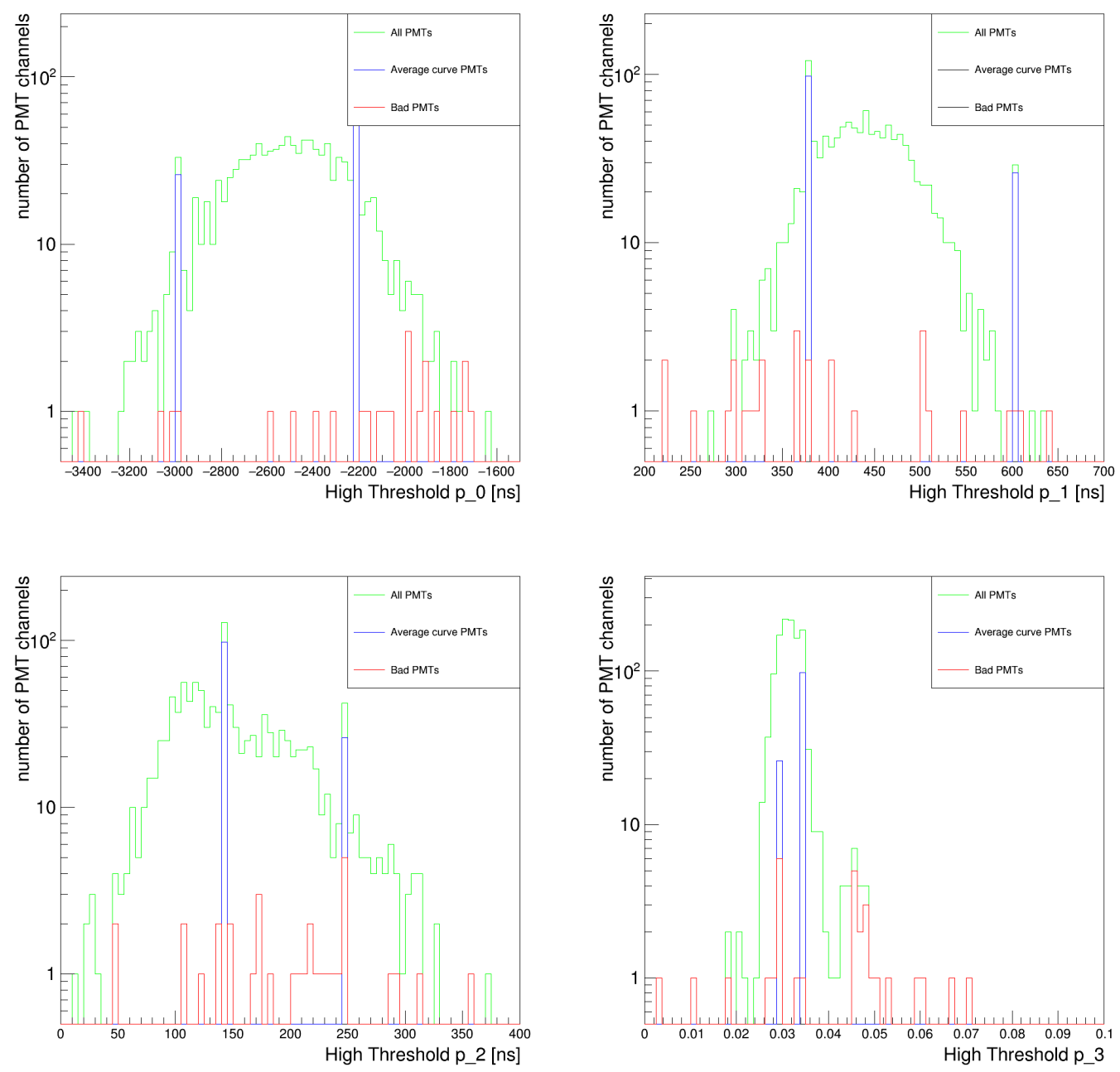

Figure 3.6: The distribution of the slewing fit parameters, $p_{0}$ (upper left), $p_{1}$ (upper right), $p_{2}$ (lower left), $p_{3}$ (lower right) for the high threshold fit. The green distribution contains all 1200 PMT IDs. The blue distribution contains all PMTs assigned the average curve, due to not being active at the time of calibration or not receiving laser light. The red distribution represents the channels known to be bad.

was taken by averaging all PMTs from the previous calibration run that passed this check [5]. First $\Delta t_{i}$, the difference between the individual and average fit at a given 
ToT value, is calculated by

$$
\Delta t_{i}\left(\text { ToT }_{i}\right)=\operatorname{Fit}\left(\text { ToT }_{i}\right)-\text { AverageFit }\left(\text { ToT }_{i}\right)
$$

and is taken to be the observed value for that ToT bin. A set of weights, $w g t_{i}$ given by,

$$
w g t_{i}(T o T)=\frac{n_{i}}{\sum_{i} n_{i}}
$$

where $n_{i}$ is the expected number of entries in each ToT bin. The weighting is done to simulate the number of data points available in each ToT bin. The weighted average difference between the fits, $\Delta t_{p m t a v e}$, is given by

$$
\Delta t_{\text {pmtave }}=\sum_{i}^{N} \Delta t_{i} * w g t_{i}
$$

is used as the expected value within a ToT bin. The difference between the individual and average fit is examined as the shape of the curve is the only thing of interest even if offset by a constant. $\chi$ is calculated by,

$$
\chi=\sqrt{\sum_{i}^{N}\left(\Delta t_{i}-\Delta t_{\text {pmtave }}\right)^{2} * w g t_{i}}
$$

which is the parameter to determine the goodness of the fit.

To determine the value of $\chi$ to cut on, the $\chi$ values for PMTs that had been previously 
flagged as bad from three runs were examined, and the lowest $\chi$ value of a bad channel was 9.53. To leave a buffer, the cut value was set to 9.00. After examining all calibration runs from before Sept. 2015, any channel that was visibly bad was caught by this check. Some of the channels that were either previously marked good or questionable had their status changed after the check.

\subsubsection{Timing Pedestal Calibration}

The timing pedestals are the offset between the arrival time of the air shower plane expected from the directional reconstruction of the air shower event which uses only the PMT channels that are hit by the event, and the signal time actually measured in an individual PMT channel participating in the reconstruction of the event. To produce these pedestals, 15,000 events with an event size exceeding 500 participating PMT channels (nHit>500) are reconstructed. The pedestal is the mean of a Gaussian fit to the residual distribution. This pedestal is added to $p_{2}$ of Eq. 3.6 and the process is repeated a total of three times, each reducing the magnitude of the pedestal value. The number of iterations is chosen such that the change in the pedestals is less than or equal to $0.1 \mathrm{~ns}$ between iterations [21]. 


\subsubsection{Timing Autocheck}

The purpose of the timing autocheck is to remove PMT channels that after completeing the calibration procedure still had a significant time residual or a time residual width much smaller or larger than is typical for the given PMT. The time residual is calculated by taking the mean of the residuals for a channel from one subrun of reconstructed data of nHit $>500$ that passes the standard cuts and angle fit cut. The time residual width is the width of the time residual distribution of a given PMT after reconstruction of data. Cuts of \pm 1.0 ns for the time residual and $\mathrm{PE}>5$ and $\mathrm{PE}>200$ were applied. Also cuts of $1.9 \mathrm{~ns}<$ residual width $<3.1 \mathrm{~ns}$ for $\mathrm{PE}>5$ and $1.0 \mathrm{~ns}<$ residual width $<2.4$ ns at $\mathrm{PE}>200$. These cuts were chosen so that they would catch PMTs that are outside of the standard distribution for each parameter. Sample distributions for the time residual and time residual width for events $>5 \mathrm{PE}$, including outliers, are shown in Fig. 3.7 [5].
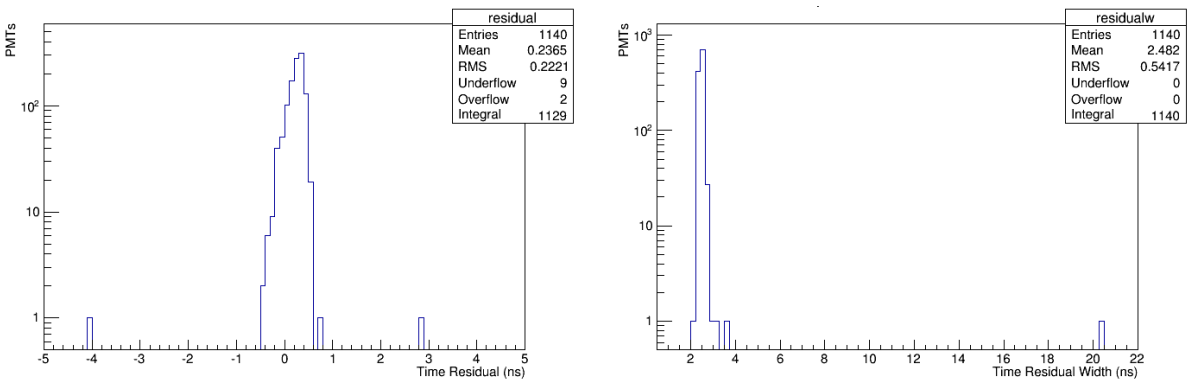

Figure 3.7: Distributions of time residuals and time residual widths for $\mathrm{PE}>5$ including outlier PMTs that would be removed by flagged as bad by timing check 
Initially, stricter cuts were applied over a large sample of data for each calibration period and if a PMT failed the cut in $10 \%$ of subruns checked, it would be marked as bad. To monitor the calibration over time this was separated into two parts: the loose cut described here and the strict cut applied in the diagnostic checks at the end of the chapter.

\subsection{Zenith Alignment}

After the time pedestals are applied the timing plane, the effective plane of the detector, is essentially flat but not always pointing straight up. There is typically $1^{\circ}$ offset for timing plane. To correct for this the apparent zenith of the detector is found and a set of additional time pedestals is created and added to $p_{2}$ of Eq. 3.6. This rotates the timing plane so that it points directly overhead. To create the pedestals, a full day of data is reconstructed using the current calibration including the bad channel lists. This rotation brings the apparent zenith to within $0.1^{\circ}$ of the actual zenith [21]. 


\subsection{Diagnostic Checks}

The last step of calibration before event reconstruction is to run the diagnostic checks over a sub-sample of data. Each sub-sample is approximately 2 weeks of data. Running over each sub-sample produces an additional bad channel list to be used for its data period. This is to catch variation within the detector over time. The timing cuts applied to the tranches are \pm 0.3 ns of the mean of a Gaussian fit for the time residuals and residual widths. This value was chosen as it is slightly more than 2 times the RMS for each distribution once outliers are removed. In addition to the strict timing check it also runs a set of checks to monitor the charge. PMTs that fail at least one cut in $10 \%$ of the samples checked are flagged as bad for that 2 weeks. 



\section{Chapter 4}

\section{Gamma/Hadron Separation with}

\section{Multivariable Analysis}

Gamma/Hadron separation is critical for the analysis of HAWC data in order to perform measurements of gamma-ray emission because the percentage of gamma-ray events in the data is about $0.1 \%$. To separate between the two types of events, the Toolkit for Multivariate Data Analysis (TMVA) with ROOT is used [11] [6]. This is a software library built on top of ROOT, both being developed by CERN. In particular the boosted decision tree (BDT) method is used for the classification of gamma-ray showers as signal, and hadron showers as background. 


\subsection{TMVA Boosted Decision Trees}

BDTs are binary trees that allow the separation of signal and background data from one another. There are two parts to creating BDTs: training and testing. Training is the actual construction of the trees based on a sample set of data where each event's actual classification is known. This produces a weights file that is used for the classification of events. Testing is using a separate set of sample data that is also known to evaluate the ability of the separation method to correctly identify known events.

\subsubsection{Training of BDTs}

The BDTs are created by taking a set of gamma rays simulated using the Cosmic Ray Simulations for KASCADE (CORSIKA) software package, as the signal data [7]. For the background, air shower events recorded by the HAWC array are used, because hadron showers are harder to simulate properly. While the recorded air showers will include gamma-ray air showers, this impurity of the background is small and does not affect the separation ability of the method. The initial data set was separated by the TMVA package automatically into a training subset and testing subset. The following are used as separation variables: PINCness, CxPE40, nHit, the age of the 
shower from the lateral distribution function $(s)$, and the amplitude of the lateral distribution function $(\log (A))$. These parameters are defined by,

$$
\log (Q)=\log (A)+s\left[\log \left(\frac{r}{R_{M o l}}\right)+\log \left(1+\frac{r}{R_{M o l}}\right)\right]-3 \log \left(\frac{r}{R_{M o l}}\right)-4.5 \log \left(1+\frac{r}{R_{M o l}}\right)
$$

where $Q$ is defined by Eq. 2.5 [16]. The parameter PINCness was chosen because it is currently used in HAWC for gamma/hadron separation. CxPE40 and nHit are contained in the compactness variable as defined by Eq. 2.8 and are also currently used for the $\mathrm{G} / \mathrm{H}$ separation. The shower age and amplitude were suggested by a colleague after it was found that these variables showed imporvement in separation strength at high $f_{\text {hit }}$ [12] [19]. A separation parameter is chosen and a cut is applied on that parameter, this process repeats each time a different separation parameter can be chosen, until one of the stopping criterion is met. The stopping criteria are: a max node depth of 10 has been reached, a node is either completely signal or background, or a node contains less than $1 \%$ of the initial data sample for that tree. The parameters for the BDTs used in the training, testing and analysis of the Crab Nebula described in this chapter are in table 4.1. The AdaBoost, bagging and randomization of trees described in Ch. 1 are used in the training of trees, meaning that for the AdaBoost the input for each tree is selected from a set of sample data with replacement and at each split only a subset of variables, 2 in this case, is considered when maximizing the separation gain. The separation index can also defined as the purity of the node as opposed to a simple \pm 1 . Events are binned into the fHit bins shown in Table 2.1 
Table 4.1

List of tree parameters, with the min node size being a percentage of initial input events in a tree. Each boosting method described in Ch. 1 is used. The leaf nodes are defined based upon their purity instead of given a simple \pm 1

\begin{tabular}{|l|l|}
\hline Number of BDTs & 800 \\
\hline Max Node Depth & 10 \\
\hline Min Node Size & $1 \%$ \\
\hline Use AdaBoost & Yes \\
\hline Use Bagging & Yes \\
\hline Use Random Forest & Yes \\
\hline Use Node Purity & Yes \\
\hline
\end{tabular}

before training, and a set of BDTs is produced for each bin.

\subsubsection{Testing of BDTs}

The testing of the BDTs allows for the examination of Monte Carlo simulation of signal efficiency vs background rejection along with determining whether the BDTs need pruning (the removal of leaf nodes) due to overtraining, and learning all of the statistical fluctuations of the training data. If overtraining occurs the test sample will have significantly lower performance than the training sample. The distribution of the response value, $y_{\text {boost }}$, defined in Eq. 1.7, for $f_{\text {hit }}$ bin 9 is shown in Fig. 4.1. The results for $f_{\text {hit }}$ bins 1-8 are shown in Appendix A.1. No significant overtraining was found in any of the nine bins. Plots of the efficiency for both the signal and background vs $y_{\text {boost }}$ for $f_{\text {hit }}$ bin 9 are shown in Fig 4.2 with the remaining bins in Appendix A.1. 


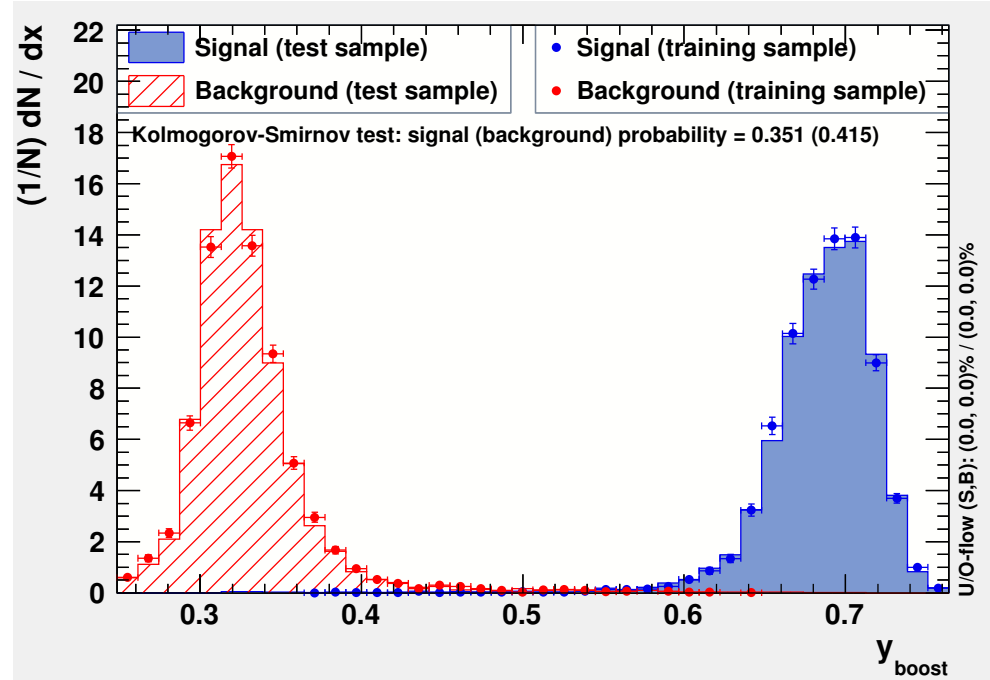

Figure 4.1: The response values, $y_{\text {boost }}$, of signal and background data for both the training and testing of the BDTs shown for $f_{\text {hit }}$ bin 9 .

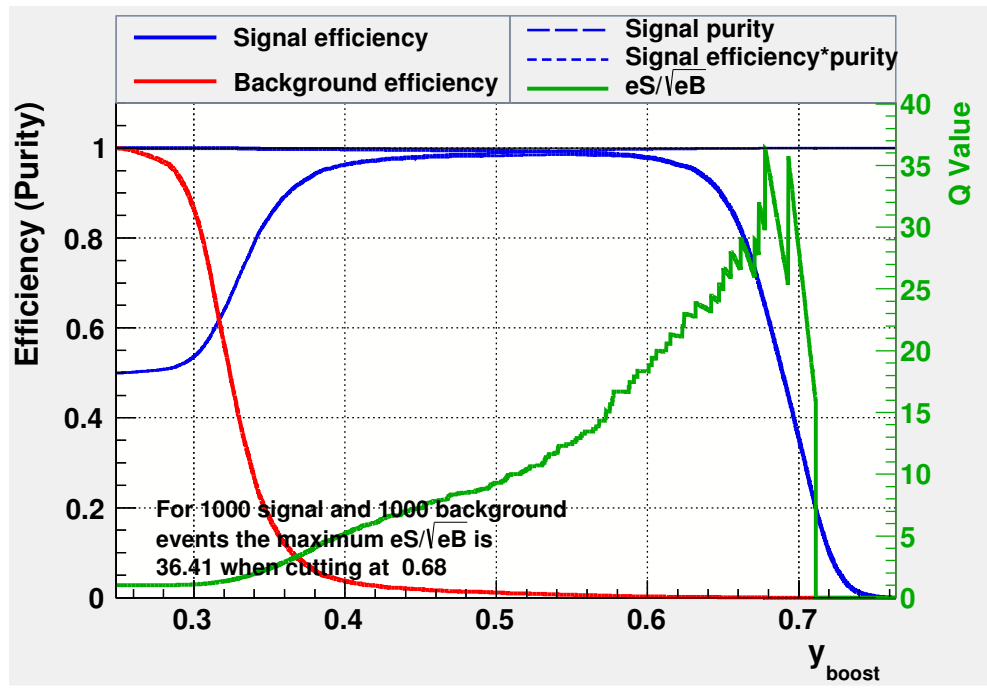

Figure 4.2: Efficiency plot for both the signal and background data vs the response value, $y_{\text {boost }}$, for $f_{\text {hit }}$ bin 9 . 


\subsection{Analysis and Results of the Crab Nebula}

To compare the new method of using BDTs to separate gamma-ray showers from hadron showers with the standard method of cutting on the parameter PINCness and Compactness individually, two months of Crab strip data, Nov-Dec, 2015, was examined. The Crab strip data consisted of events that are within $\pm 3^{\circ}$ of the Crab Nebula in declination, and within $40^{\circ}$ of the Crab Nebula in right ascension. The events were binned, and then processed with the classification algorithm of TMVA which produced response values from zero (background-like) to one (signal-like) for each event. As the PINCness and Compactness cuts were optimized to maximize the significance on the Crab Nebula in each $f_{h i t}$ bin, maximizing the significance on the Crab Nebula in each $f_{\text {hit }}$ was also used for the response value cut.

Significance maps were created using the method of direct integration to calculate the background [1]. Direct integration assumes, for 2 hour blocks of time, the all-sky rate is independent of the spatial arrival distribution of events in the detector. The background at any point in the sky, during this time, is determined by convolving the all-sky rate with the spatial distribution of events in detector coordinates. When calculating the background a $1.3^{\circ}$ radius region centered on the Crab Nebula was masked and not used in the background calculation. The background was smoothed by averaging all points with in a $0.5^{\circ}$ radius, as some spatial bins are empty or near 
empty at high $f_{\text {hit }}$ values. The response value distribution was cut on successively in 0.01 intervals to find the maximum significance value for each bin. Also, a power law with a spectral index of 2.63 and a pivot energy of $7 \mathrm{TeV}$ were assumed. These maps used an detector response file optimized for PINCness and Compactness cuts to obtain initial cut values to produce a new detector response file optimized on cuts for $y_{\text {boost }}$. Then the maps were recreated with the new detector response file and the new maximum significance cuts were found. The standard cuts on PINCness and Compactness were applied for each bin, and significance maps for those were produced as well. These were then compared with one another. To calculate the significance values the maximum likelihood method was used to calculate a test statistic (TS), which indicates how likely the observations are to be background only or due to combined background and point source emission. This uses pixels within $2^{o}$ of the Crab Nebula. The angular resolution of the Crab Nebula is defined by the point spread function, a measure of how accurately the photon direction is reconstructed. The angular resolution is worse in lower $f_{\text {hit }}$ bins (1-3) causing the size of the Crab Nebula and therefore the area needed to perform the likelihood method to increase [1]. For these low $f_{\text {hit }}$ bins this area extends past the upper and lower bounds of the declination band for the data set used in this study causing the likelihood method to fail. Hence, the significance maps for these bins are not meaningful. The significance map for the PINCness and Compactness cuts and the $y_{\text {boost }}$ cut for $f_{\text {hit }}$ bin 9 are shown in Fig 4.3. The comparisons of the standard and BDT significance maps for 

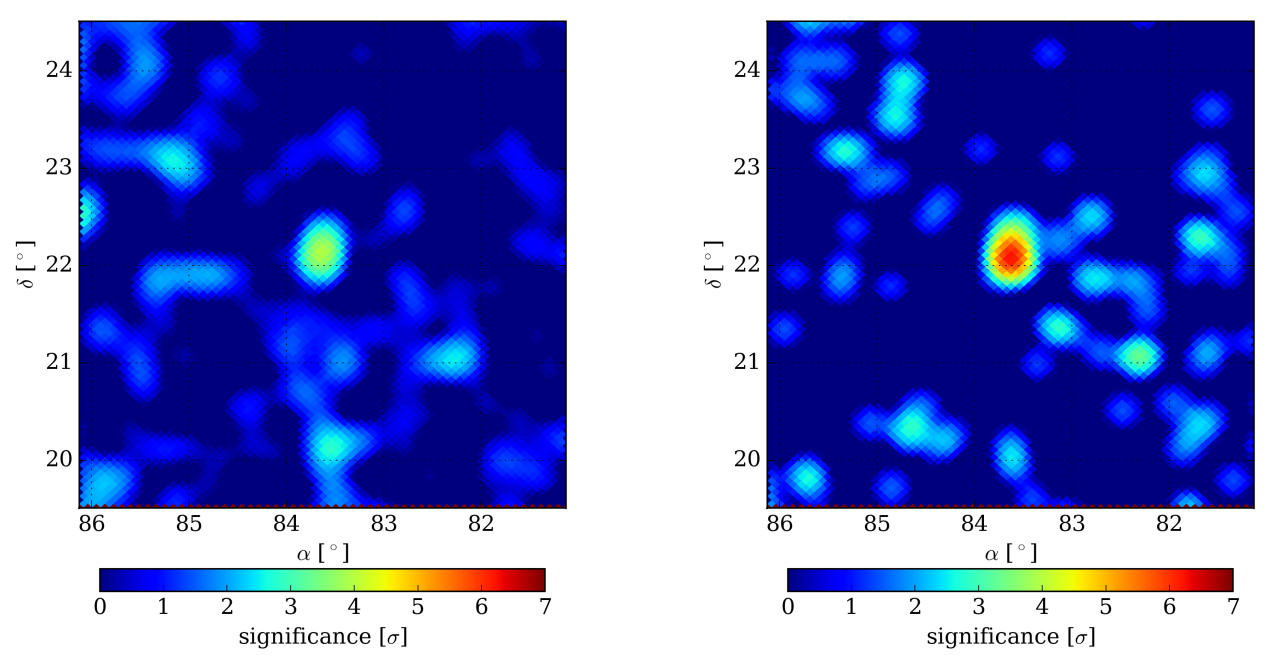

Figure 4.3: The $f_{\text {hit }}$ bin 9 significance maps of the Crab Nebula for NovDec 2015 for both the standard HAWC cuts (left) and the response value cut (right)

$f_{\text {hit }}$ bins 4-8 are shown in Appendix A.2.

In Table 4.2, the efficiencies for gamma-ray and hadron showers and the maximum significance for $f_{h i t}$ bins 4-9 for both the standard cuts and the response value cut along with the cut value on the response value are reported. The efficiencies for the response value cuts are from the TMVA testing. For $f_{\text {hit }}$ bins 6-9 the maximum significance increased by at least 0.89 sigma with the largest increase of 2.56 sigma in $f_{\text {hit }}$ bin 9 . The gamma ray efficiency in bins 4-9 increased as well. Though the background rejection decreased in all but bin 6 , in bins 7-9 the decrease was small. 
Table 4.2

The gamma ray and hadron efficiencies and the maximum significance value for $f_{\text {hit }}$ bins 4-9 for both the standard PINCness and compactness cuts and the response value cut. The cut value for the response value yielding the maximum significance is also shown for each bin. The gamma and hadron efficiencies for the standard cuts are taken from the published HAWC analysis of the Crab Nebula [1] and the efficiencies for the response value are from the testing results.

\begin{tabular}{|c|c|c|c|c|c|c|c|}
\cline { 2 - 8 } \multicolumn{1}{c|}{} & \multicolumn{3}{c|}{ Standard Cuts } & \multicolumn{4}{c|}{ Classifer Response } \\
\hline Bin\# & $\epsilon_{\gamma}$ & $\epsilon_{h}$ & $\max \sigma$ & $\epsilon_{\gamma}$ & $\epsilon_{h}$ & $\max \sigma$ & cut value \\
\hline 4 & 51 & 1.3 & 14.53 & 95 & 2.5 & 13.94 & 0.51 \\
\hline 5 & 50 & 0.55 & 12.44 & 80 & 7.9 & 12.10 & 0.53 \\
\hline 6 & 35 & 0.21 & 10.23 & 65 & 0.11 & 11.12 & 0.53 \\
\hline 7 & 63 & 0.24 & 8.24 & 90 & 0.47 & 9.19 & 0.58 \\
\hline 8 & 63 & 0.13 & 6.84 & 95 & 0.46 & 8.86 & 0.61 \\
\hline 9 & 70 & 0.20 & 3.67 & 98 & 0.38 & 6.23 & 0.64 \\
\hline
\end{tabular}





\section{Chapter 5}

\section{Conclusion}

\section{$5.1 \quad$ Summary}

\subsubsection{Calibration}

The calibration checks have allowed for a more systematic set of checks and do not depend upon an individual manually calibrating the detector to determine the quality of the calibration. Still improvements are necessary and individual checks are not superfluous. The calibration code is currently being updated, and portions of the checks presented in this thesis will be replaced. In particular the slewing curve check will likely be replaced with a simple $\chi^{2}$ check because the data points will be saved 
and available. The timing check needs further study on how strict the timing cut should be, since a strict cut is being applied over two week periods. The calibration checks provided an initial diagnostic tool that can be run quickly to determine if channels are not sufficiently calibrated and the presented procedure is a first step to improve the calibration of the HAWC observatory.

\subsubsection{Gamma Hadron Separation with BDTs}

Results from Ch. 4, where the BDT analysis was applied to the region of the Crab Nebula, show significant promise that using BDTs to separate gamma-ray signal from hadronic background data can improve the sensitivity of the analysis to gamma rays. The analysis of events with highest $f_{\text {hit }}$ values (bins 6-9), the events with a very bright shower footprint in the HAWC array, showed improvement in significance over the current default analysis of HAWC data using individual quality cuts on PINCness and compactness. It is quite possible that with further optimization the performance for

intermediate $f_{\text {hit }}$ values (bins 4 and 5) could be improved. Another approach would be to apply the BDT style analysis only to the events with high $f_{\text {hit }}$ values. 


\subsection{Continuing Work}

\subsubsection{Calibration Procedure Update}

As stated above the calibration code is being updated. The goals of the update are to streamline the procedure and combine the various pieces of code into a single package. Because the individual data points for all timing and charge curves will be saved in the future once this update is complete, a simple $\chi^{2}$ method can be used to compare individual curves for a new calibration run with the curves of the previous calibration run to detect any changes in an individual PMT channel.

\subsubsection{Gamma Hadron Separation Optimization}

Further study into the performance of BDTs for separating gamma and hadron showers are necessary. Particularly need are more comprehensive studies on the properties of the BDTs including: number of trees, tree depth, separation variables, and boosting methods. For $f_{\text {hit }}$ bins 1-3 a larger data set should be used so that the background can be properly calculated using direct integration. A different signal weighting for the BDTs should be investigated because the assumption in the presented study was an isotropic set of gamma-ray events for both training and testing. Transit source 
weighting may allow for better rejection and should be examined. 


\section{References}

[1] Abeysekara, A. U., Albert, A., Alfaro, R., et al. 2017, apj, 843, 39

[2] Aharonian, F., Buckley, J., Kifune, T., \& Sinnis, G. 2008, Reports on Progress in Physics, 71, 096901

[3] Ayala Solares, H. 2017, PhD thesis, Michigan Technological University

[4] Bartoli, B., Bernardini, P., Bi, X. J., et al. 2013, apj, 779, 27

[5] Braun, J., Capistrán, T., García-González, J. A., et al. 2016, HAWCMEMO

[6] Brun, R., \& Rademakers, F. 1997, Nucl. Inst. Meth. in Phys. Res. A, 81

[7] D. Heck, J. Knapp, J. N. C. G. S., \& Thouw, T. 1998

[8] Hamamatsu. 2012, Hamamatsu R5912 PMT

[9] —. 2012, Hamamatsu R7081 PMT

[10] Hampel, Z. 2017, PhD thesis, University of Wisconsin-Madison 
[11] Hoecker, A., Speckmayer, P., Stelzer, J., et al. 2007, ArXiv Physics e-prints, physics/0703039

[12] John, P. 2017, HE Background Separation, Tech. rep., Data and Algorithms call, HAWC Collaboration

[13] Kang, D. 2017, ArXiv e-prints, arXiv:1702.08743

[14] Kelley-Hoskins, N. C. 2012, Master's thesis, Michigan Technological University

[15] Luna. 2012, Optical Backscatter Reflectometer ${ }^{\mathrm{TM}}$ (Model OBR 4600)

[16] Malone, K. 2015, Documention for AERIE module LatDist, Tech. rep., Pennsylvania State University

[17] Medina, B., \& Younk, P. 2016, Measurements of Calibration Fibers at HAWC with a LUNA Optical Backscatter Reflectometer, Tech. rep., Physics Division, Los Alamos National Laboratory

[18] Photonics, T. 2012, PNG Lasers by Teem Photonics

[19] Pretz, J. 2017, personal communication

[20] Products, S. 2012, Spectral Products AB301-T Filter Wheel

[21] Solares, H. A., Gerhardt, M., Hui, C. M., et al. 2016, PoS, ICRC2015, 997

[22] Wood, J. 2016, PhD thesis, University of Maryland 
[23] Zhou, H. 2015, 12 Steps to Perfect Timing Calibration, Tech. rep., Michigan Technological University

[24] —. 2015, PhD thesis, Michigan Technological University 



\section{Appendix A}

\section{Plots from Multivariate Analysis}

\section{A.1 Training and Testing Plots for bins 1-8}
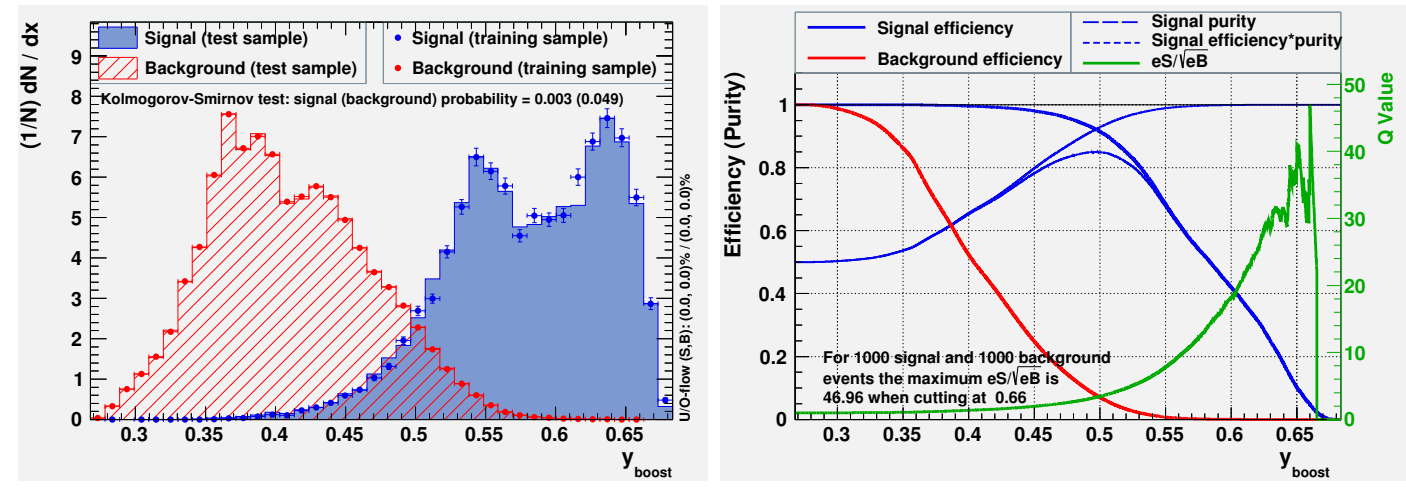

Figure A.1: Response values for the BDTs for signal and background testing and training data (left) and effiecieny of the background and signal data vs response value(right) using $f_{\text {hit }}$ bin 1 . 

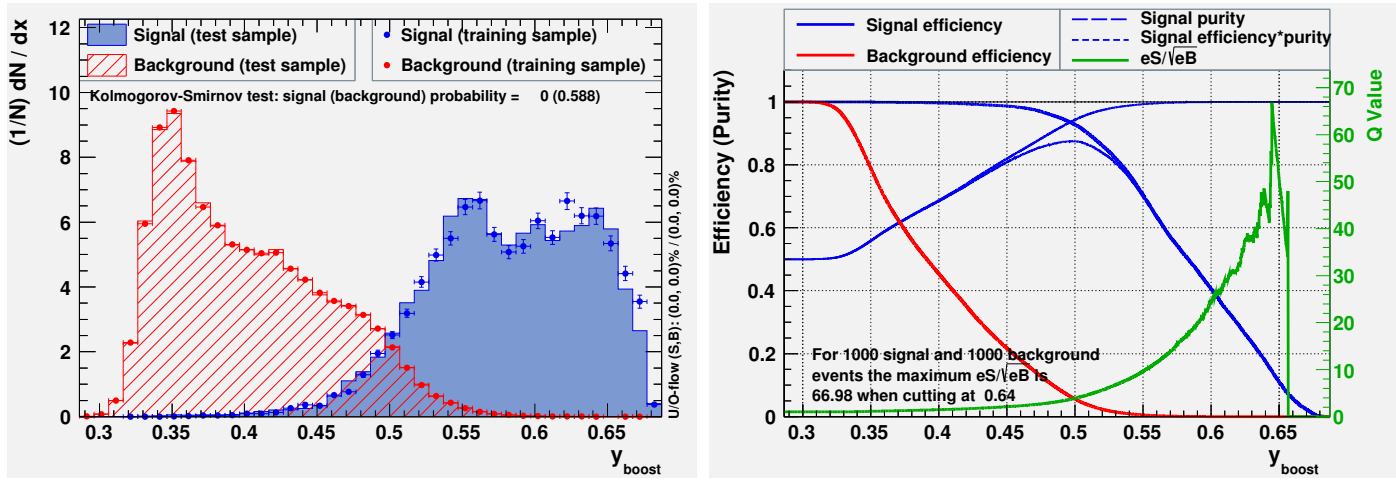

Figure A.2: Response values for the BDTs for signal and background testing and training data (left) and effiecieny of the background and signal data vs response value (right) using $f_{\text {hit }}$ bin 2 .
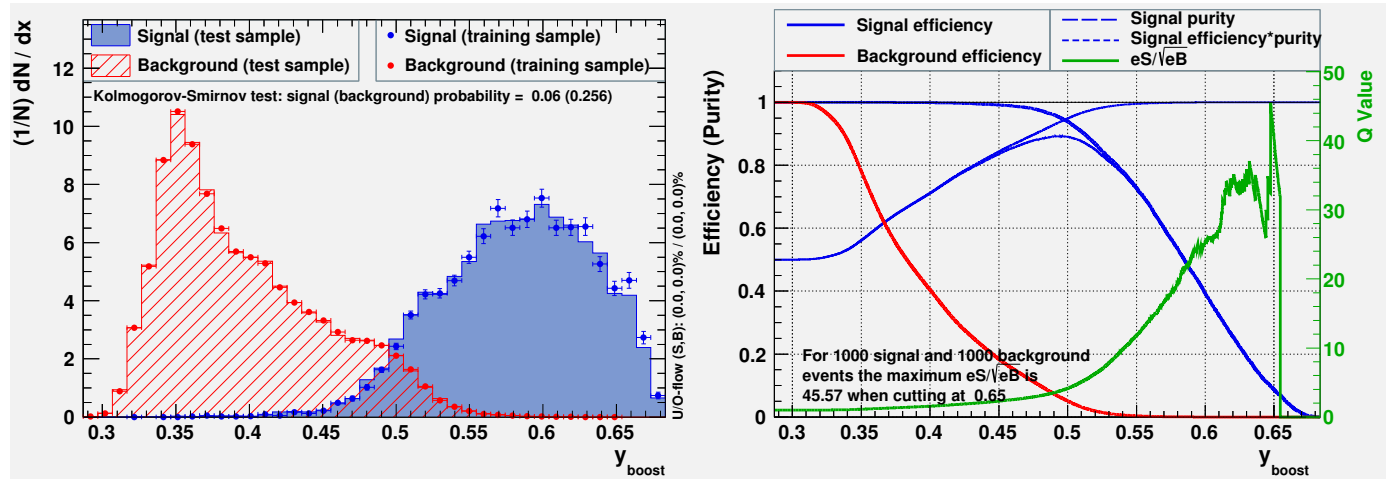

Figure A.3: Response values for the BDTs for signal and background testing and training data (left) and effiecieny of the background and signal data vs response value (right) using $f_{\text {hit }}$ bin 3 . 

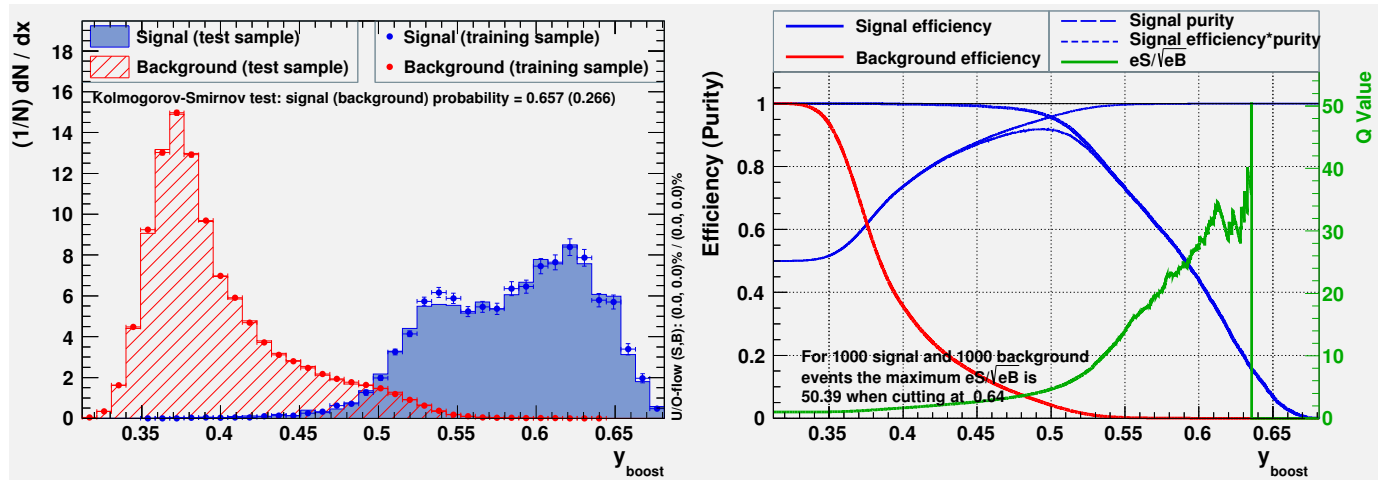

Figure A.4: Response values for the BDTs for signal and background testing and training data (left) and effiecieny of the background and signal data vs response value (right) using $f_{\text {hit }}$ bin 4 .
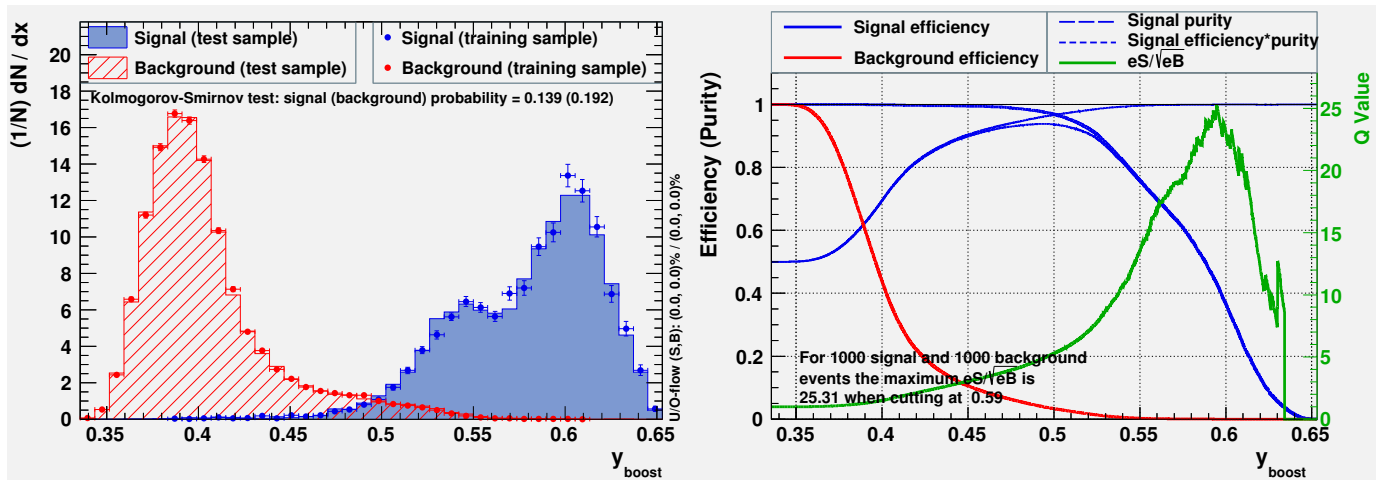

Figure A.5: Response values for the BDTs for signal and background testing and training data (left) and effiecieny of the background and signal data vs response value (right) using $f_{\text {hit }}$ bin 5 .
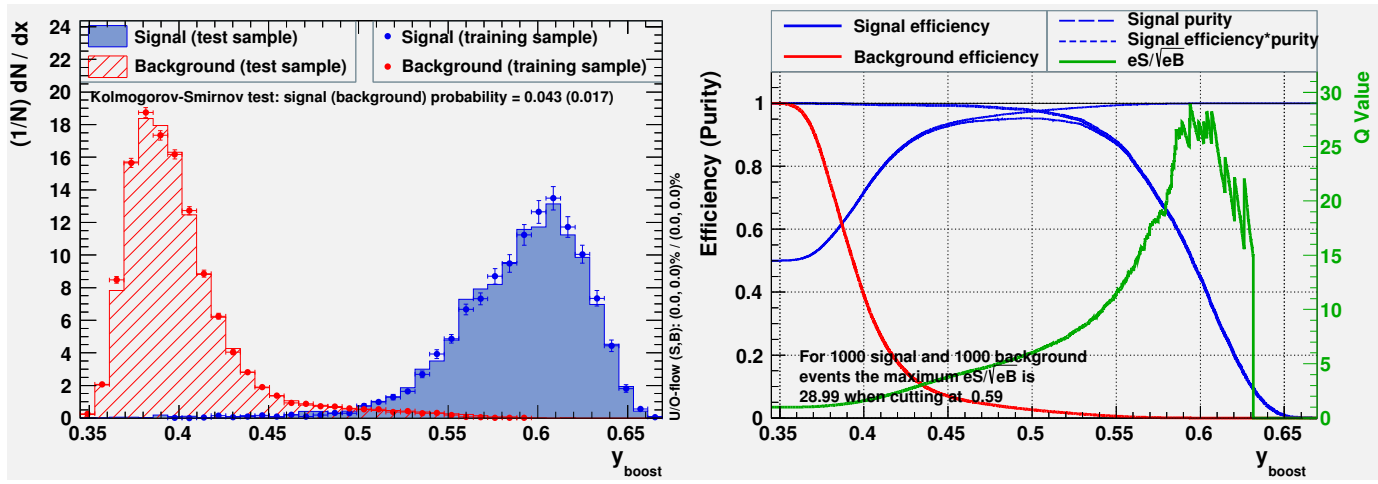

Figure A.6: Response values for the BDTs for signal and background testing and training data (left) and effiecieny of the background and signal data vs response value (right) using $f_{\text {hit }}$ bin 6 . 

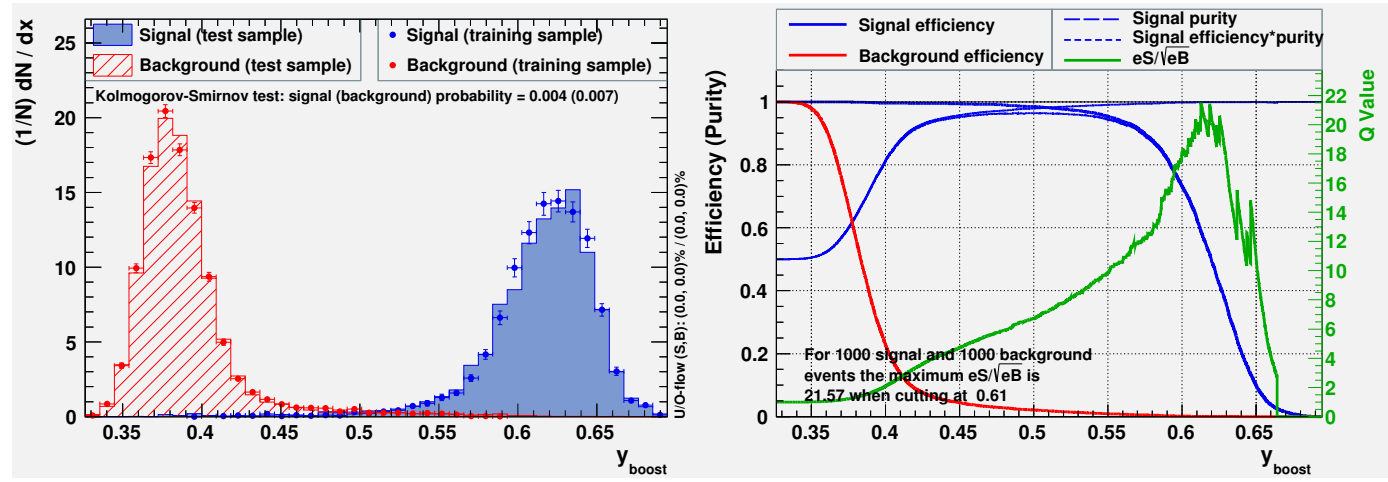

Figure A.7: Response values for the BDTs for signal and background testing and training data (left) and effiecieny of the background and signal data vs response value (right) using $f_{\text {hit }}$ bin 7 .
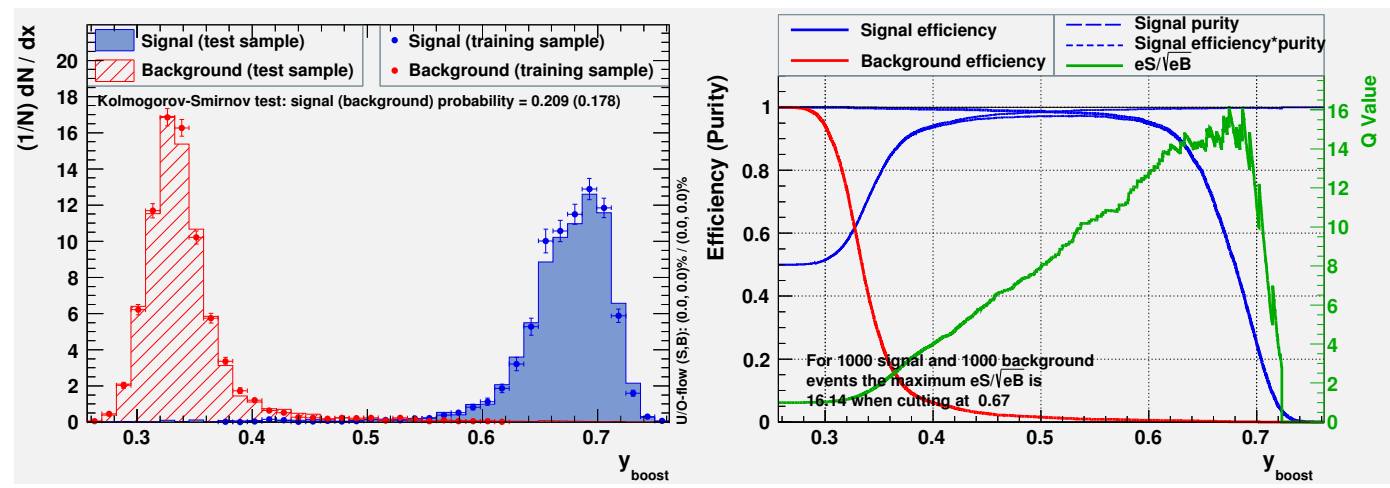

Figure A.8: Response values for the BDTs for signal and background testing and training data (left) and effiecieny of the background and signal data vs response value (right) using $f_{\text {hit }}$ bin 8 .

\section{A.2 Crab Significance Maps for bins 4-8}



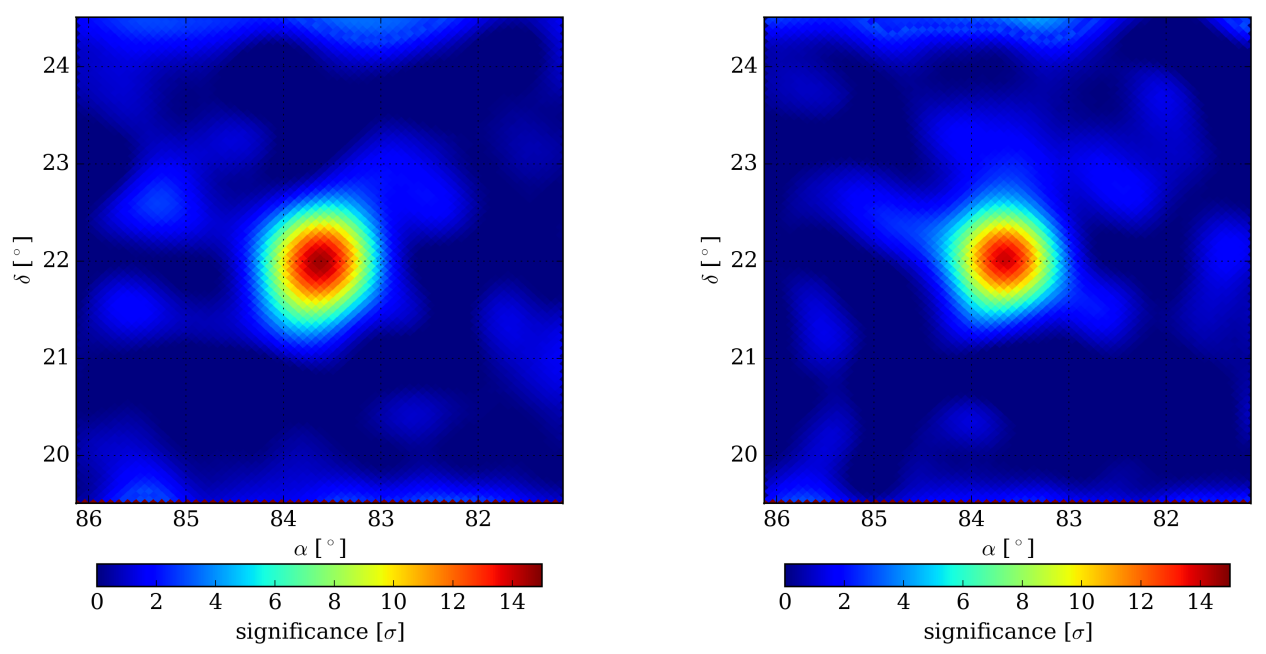

Figure A.9: Significance maps of the Crab Nebula for $f_{\text {hit }}$ Bin 4 with standard PINCness and compactness cuts (left) and new BDT response value cuts (right)
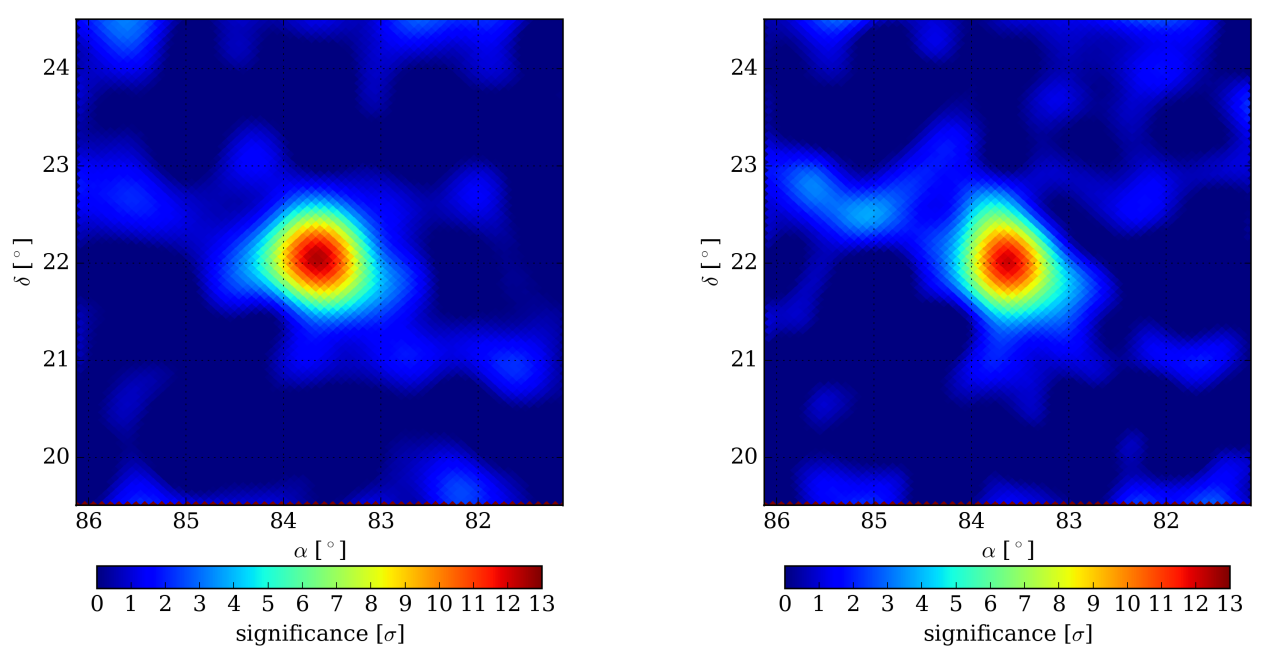

Figure A.10: Significance maps of the Crab Nebula for $f_{\text {hit }}$ Bin 5 with standard PINCness and compactness cuts (left) and new BDT response value cuts (right) 

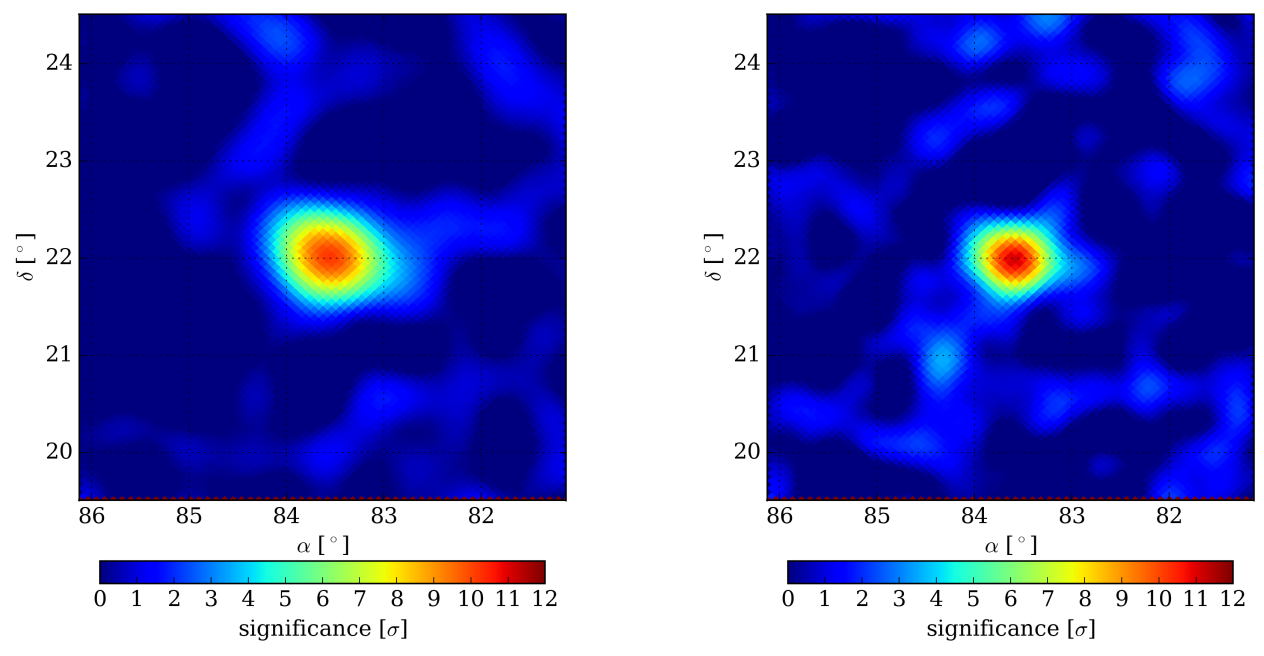

Figure A.11: Significance maps of the Crab Nebula for $f_{\text {hit }}$ Bin 6 with standard PINCness and compactness cuts (left) and new BDT response value cuts (right)
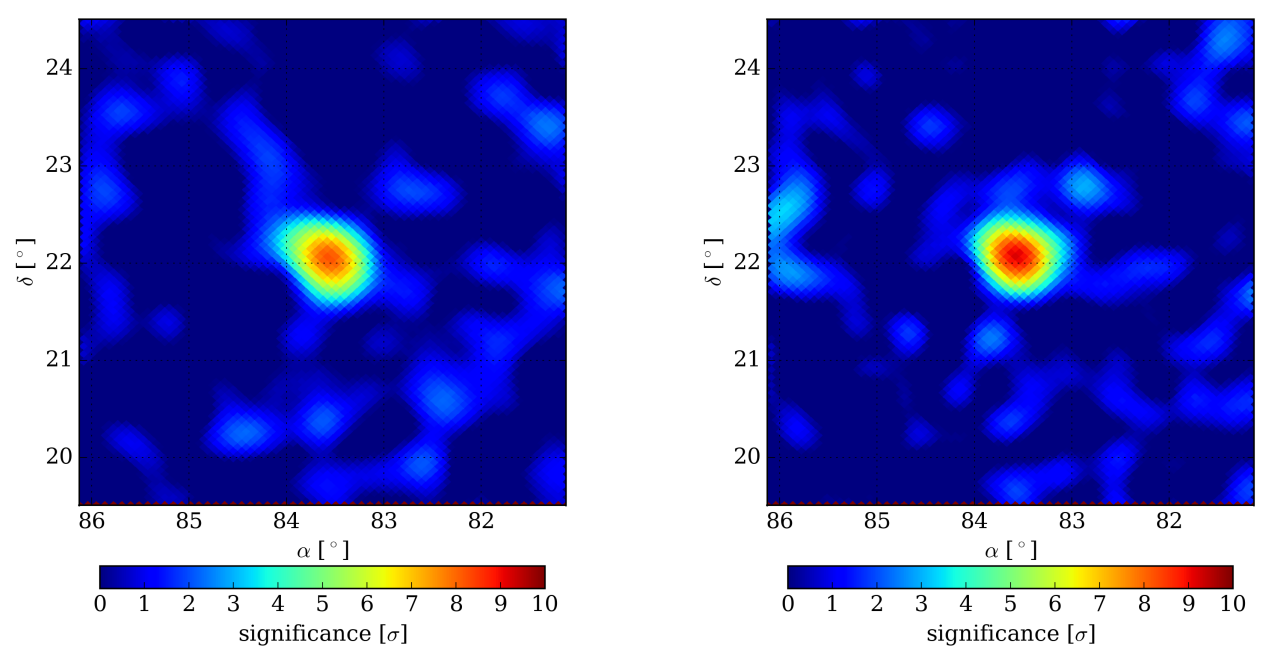

Figure A.12: Significance maps of the Crab Nebula for $f_{\text {hit }}$ Bin 7 with standard PINCness and compactness cuts (left) and new BDT response value cuts (right) 

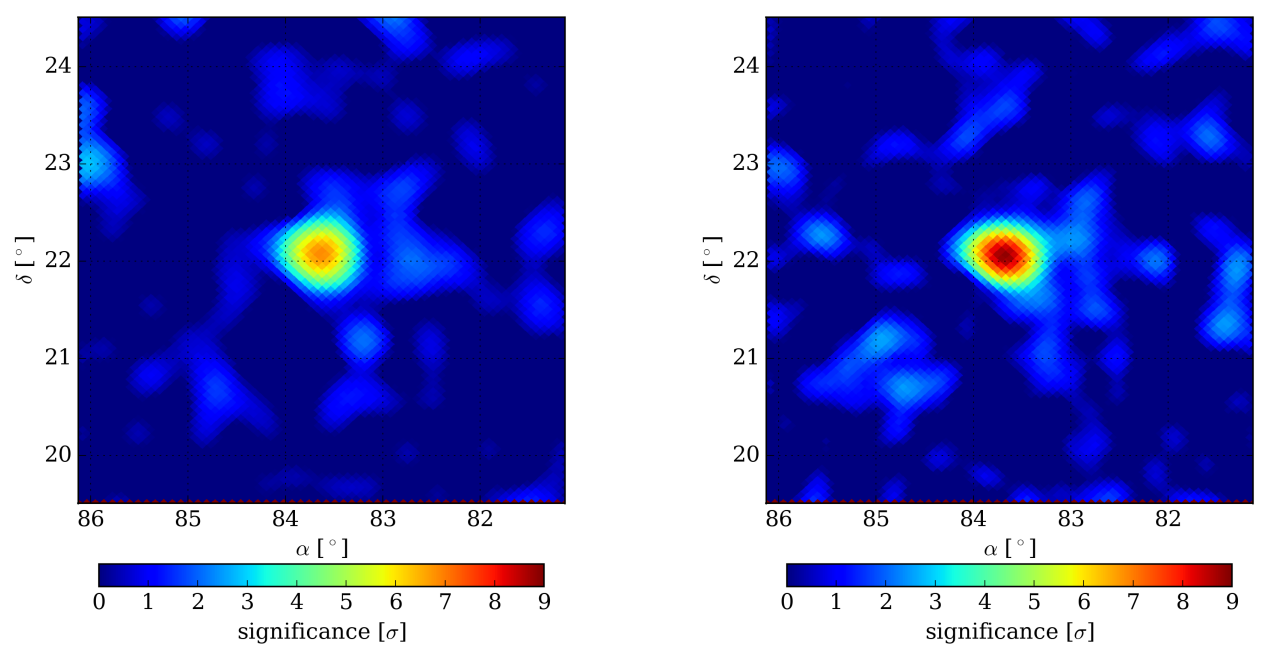

Figure A.13: Significance maps of the Crab Nebula for $f_{\text {hit }}$ Bin 8 with standard PINCness and compactness cuts (left) and new BDT response value cuts (right) 


\section{Appendix B}

\section{Letters of Permission}

B.1 Figure 1.2

Image credit: Zigfried Hampel. Permission granted by private communication.

B.2 Figure 1.4

Image credit: Hugo Ayala Solares. Permission granted by email. 
B.3 Figure 2.1

Image credit: HAWC Collaboration

B.4 Figure 2.2

Image credit: Zigfried Hampel. Permission granted by private communication.

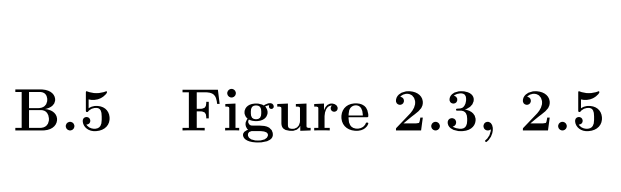

Image credit: Hao Zhou. Permission granted by email.

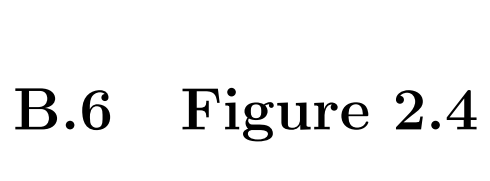

Image credit: Hugo Ayala Solares. Permission granted by email. 
B.7 Figure 2.7

Image credit: HAWC Collaboration. Retrieved from [14].

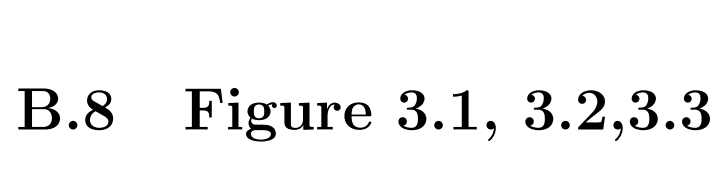

Image credit: Zhixiang Ren. Permission granted by private communication. 